\title{
Synthesis of Both Enantiomers of a P-Chirogenic 1,2-Bisphospholanoethane Ligand via Convergent Routes and Application to Rhodium Catalyzed Asymmetric Hydrogenation of CI-1008 (Pregabalin)
}

\author{
Garrett Hoge \\ Contribution from Pfizer Global Research and Development, \\ 2800 Plymouth Road, Ann Arbor, Michigan 48105.
}

\section{Supporting Information}

Nuclear Magnetic Resonance. $400 \mathrm{MHz}{ }^{1} \mathrm{H}$ NMR, $100 \mathrm{MHz}{ }^{13} \mathrm{C}$ NMR, and $162 \mathrm{MHz}{ }^{31} \mathrm{P}$ NMR spectra were obtained on "Barton" - a Varian Unity+400 (Inova400 after 08/15/00) spectrometer equipped with an Auto Switchable 4-Nuclei PFG probe, two RF channels, and a SMS-100 sample changer by Zymark. Spectra were generally acquired near room temperature $\left(21^{\circ} \mathrm{C}\right)$, and standard autolock, autoshim and autogain routines were employed. Samples are usually spun at $20 \mathrm{~Hz}$ for 1D experiments. ${ }^{1} \mathrm{H}$ NMR spectra were acquired using 45-degree tip angle pulses, 1.0 second recycle delay, and 16 scans at a resolution of $0.25 \mathrm{~Hz} /$ point. The acquisition window was typically $8000 \mathrm{~Hz}$ from +18 to $-2 \mathrm{ppm}$ (Reference TMS at $0 \mathrm{ppm}$ ), and processing was with $0.2 \mathrm{~Hz}$ line broadening. Typical acquisition time is 80 seconds. Regular ${ }^{13} \mathrm{C}$ NMR spectra were acquired using 45-degree tip angle pulses, 2.0 second recycle delay, and 2048 scans at a resolution of $1 \mathrm{~Hz} /$ point. Spectral width was typically $25 \mathrm{KHz}$ from +235 to $-15 \mathrm{ppm}$ (Reference TMS at $0 \mathrm{ppm}$ ). Proton decoupling was applied continuously, and $2 \mathrm{~Hz}$ line broadening was applied during processing. Typical acquisition time is 102 minutes. ${ }^{31} \mathrm{P}$ NMR spectra were acquired using 45-degree tip angle pulses, 1.0 second recycle delay, and 64 scans at a resolution of $2 \mathrm{~Hz} /$ point. Spectral width was typically $48 \mathrm{KHz}$ from +200 to $-100 \mathrm{ppm}$ (Reference $85 \%$ Phosphoric Acid at 0 ppm). Proton decoupling was applied continuously, and $2 \mathrm{~Hz}$ line broadening was applied during processing. Typical acquisition time is 1.5 minutes. 
Mass Spectrometry. Mass Spectrometry was performed on a Micromass Platform LC system operating under MassLynx and OpenLynx open access software. The LC was equipped with a HP1100 quaternary LC system and a Gilson 215 liquid handler as an autosampler. Data was acquired under atmospheric pressure chemical ionization with 80:20 acetonitrile/water as the solvent. Temperatures: probe was $450^{\circ} \mathrm{C}$, source was $150^{\circ} \mathrm{C}$. Corona discharge was $3500 \mathrm{~V}$ for positive ion and $3200 \mathrm{~V}$ for negative ion.

High Performance Liquid Chromatography. High Performance Liquid Chromatography (HPLC) was performed on a series 1100 Hewlett Packard (now Agilent Technologies) instrument equipped with a manual injector, quaternary pump, and a UV detector. The LC was PC controlled using HP Chemstation Plus Software. Reverse phase HPLC was performed with a 150 x 4.6mm BDS-HypersilC18 column supplied by Keystone Scientific Incorporated. Reverse phase chiral HPLC was performed using a Chiracel OJ-R column supplied by Chiral Technologies. Normal Phase chiral HPLC was performed using Chiracel OJ, Chiracel OD, Chiracel OD-H, Chiracel AD, and Chiracel AS columns supplied by Chiral Technologies.

Gas Chromatography. Gas Chromatography (GC) was performed on a 110 volt Varian Star 3400 equipped with an FID detector with electrometer, a model 1061 packed/530 micron ID flash injector, a model 1077 split/splitless capillary injector, a relay board that monitors four external events, and an inboard printer/plotter. Gas chromatography was performed using 40m x 0.25mm Chiraldex G-TA or BTA columns supplied by Advanced Separation Technologies, Incorporated or on a $25 \mathrm{~m} \mathrm{x} .25 \mathrm{~mm}$ Coating CP Chirasil-Dex DB column supplied by Chrompack.

General procedure for asymmetric hydrogenation reactions. Pre-catalyst 36 (.01 mmole) was dissolved in $1 \mathrm{~mL}$ of methanol in a Griffin-Worden pressure vessel equipped with the attachments 
necessary to connect to a lecture bottle. The substrate $(1 \mathrm{mmole})$ was dissolved in $3 \mathrm{~mL}$ of the same solvent and this was delivered to the catalyst solution via syringe. The solution was freeze-pump-thawdegassed for three cycles with $\mathrm{N}_{2}$ and then the reaction solution was warmed to $25^{\circ} \mathrm{C}$. The vessel was then sealed and pressurized to the appropriate pressure of $\mathrm{H}_{2}$. Reaction completion times and enantiomeric excesses were monitored by chiral GC or HPLC.

Enantiomeric Excess Determinations. Enantiomeric excesses of acrylic acid derivatives were determined as follows: N-acetylalanine methyl ester (Capillary GC, Chrompack Chiral-L-Val (25 m), $120^{\circ} \mathrm{C}$, isothermal, $(\mathrm{R}) \mathrm{t}_{1}=10.5 \mathrm{~min} ;(\mathrm{S}) \mathrm{t}_{2}=11.0 \mathrm{~min} ; \mathbf{N}$-acetylphenylalanine methyl ester (HPLC, Daicel Chiralcel OJ, $1.0 \mathrm{~mL} / \mathrm{min}, 10 \%$ isopropanol/hexane, $\left.(\mathrm{R}) \mathrm{t}_{1}=11.6 \mathrm{~min} ;(\mathrm{S}) \mathrm{t}_{2}=17.7 \mathrm{~min}\right)$; $\mathbf{N}$ acetylphenylalanine and $\mathbf{N}$-acetylalanine were converted to their corresponding methyl esters via treatment with trimethylsilyldiazomethane and their enantiomeric excesses were analyzed as described above.

The CI-1008 precursors, 41 and 42, were acidified in their methanolic hydrogenation solutions with 1 $\mathrm{N} \mathrm{HCl}$. The organics were extracted into $\mathrm{CH}_{2} \mathrm{Cl}_{2}$. After drying over $\mathrm{MgSO}_{4}$ the volatiles were removed invacuo. The carboxylic acids were then converted to their corresponding methyl esters via treatment with trimethylsilyldiazomethane. The methyl esters were analyzed directly (Capillary GC, Astec GTA $(30 \mathrm{~m}), 140^{\circ} \mathrm{C}$, isothermal, $\left.(\mathrm{R}) \mathrm{t}_{1}=8.8 \mathrm{~min} ;(\mathrm{S}) \mathrm{t}_{2}=9.5 \mathrm{~min}\right)$.

Absolute Configurations. Absolute configurations of the products of hydrogenation were determined by comparison of the signs of optical rotation with those of literature values: (S)-N-Acetylalanine methyl ester $[\alpha]_{\mathrm{D}}{ }^{20}=-91.7^{\mathrm{o}}\left(\mathrm{c} 2, \mathrm{H}_{2} \mathrm{O}\right) ;{ }^{\square}(\mathbf{S})$ - $\mathrm{N}$-acetylphenylalanine methyl ester $[\alpha]_{\mathrm{D}}{ }^{20}=+16.4^{\mathrm{o}}(\mathrm{c} 2$, $\mathrm{MeOH})$ ?

The CI-1008 precursors, 41 and 42, were compared in terms of elution order to an authentic sample with (S) configuration via the method for enantiomeric excess determination (above). 


\section{Proof of relative and absolute stereochemistry of (1S,2R))-1-((-)-menthoxy)-2-}

benzylphospholaneborane (23). Although the ideal method for determining the relative and absolute stereochemistry of $\mathbf{2 3}$ was via x-ray crystallography, we were unsuccessful at growing crystals of this compound. We also attempted ${ }^{1} \mathrm{H}$ NMR NOE difference spectra to attempt to find NOE interaction between $\mathrm{H}_{\mathrm{a}}$ and $\mathrm{H}_{\mathrm{b}}$ (Figure 1S). If NOE were observed between $\mathrm{H}_{\mathrm{a}}$ and $\mathrm{H}_{\mathrm{b}}$ we could conclude that the benzyl substituent on the phospholane ring is cis relative to the borane group. However, we failed to see NOE between the two protons.

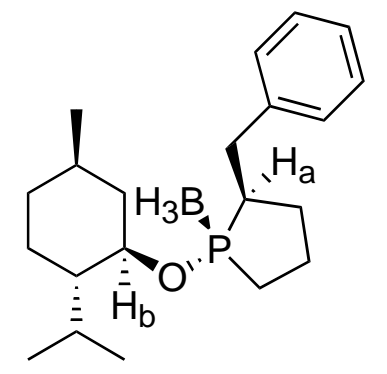

23

Figure 1S. Protons $\mathrm{H}_{\mathrm{a}}$ and $\mathrm{H}_{\mathrm{b}}$ of compound 23.

There is precedent for alkylations of (1)-alkoxyphospholaneoxides to occur cis relative to the oxide when the alkoxy group attached to phosphorous is large. stereochemistry of $\mathbf{2 3}$, but not proof.

We decided to synthesize a derivative of $\mathbf{2 3}$ using the same chiral alkylation method except using carbon dioxide gas as an electrophile rather than benzyl bromide (Scheme 1S). 
Scheme 1S. Synthesis of carboxylic acid $43{ }^{\text {a }}$

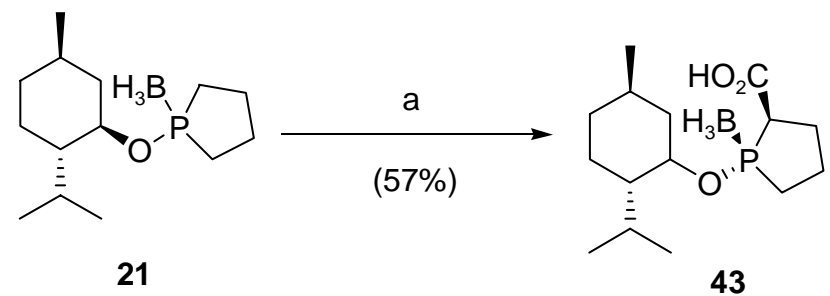

${ }^{\text {a }}$ Reagents and conditions: a) 1. s-BuLi, (-)-Sparteine, THF, $-78^{\circ} \mathrm{C} 2 . \mathrm{CuCl}_{2} 3 . \mathrm{HCl}$.

This carboxylic acid derivative was a white solid and we were able to grow x-ray quality crystals for crystallography to confirm the structure. Examination of the x-ray crystal structure of $\mathbf{4 3}$ lead us to the stereochemical assignment of $\mathbf{2 3}$ by analogy.

\section{Proof of absolute and relative stereochemistry of (1S,2R)-2-benzyl-1-methyl-phospholane (20).} Having assigned the relative and absolute stereochemistry of $\mathbf{2 3}$ and comparing the structure to that of rhodium complex 36, the absolute and relative stereochemistry of $\mathbf{2 0}$ can be assigned as drawn in Figure 2S. This information also served as evidence that the MeLi displacement of the menthoxy group of $\mathbf{2 3}$ to form $\mathbf{2 0}$ proceeded with retention of configuration at the phosphorous atom.

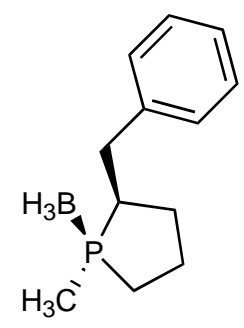

20

Figure 2S. Relative and absolute stereochemical assignment of 20.

In addition to this proof, we performed ${ }^{1} \mathrm{H}$ NMR NOE difference spectra on 20 attempting to find NOE interaction between the methyl group protons (a) and the methine proton (b) on the phospholane 
ring (Figure 3S). We observed significant NOE between the methyl protons (a) and the methine proton (b) while irradiating at the methyl proton frequency. This experiment provided additional evidence that the methine proton is cis with respect to the methyl group on the phospholane ring of $\mathbf{2 0}$.
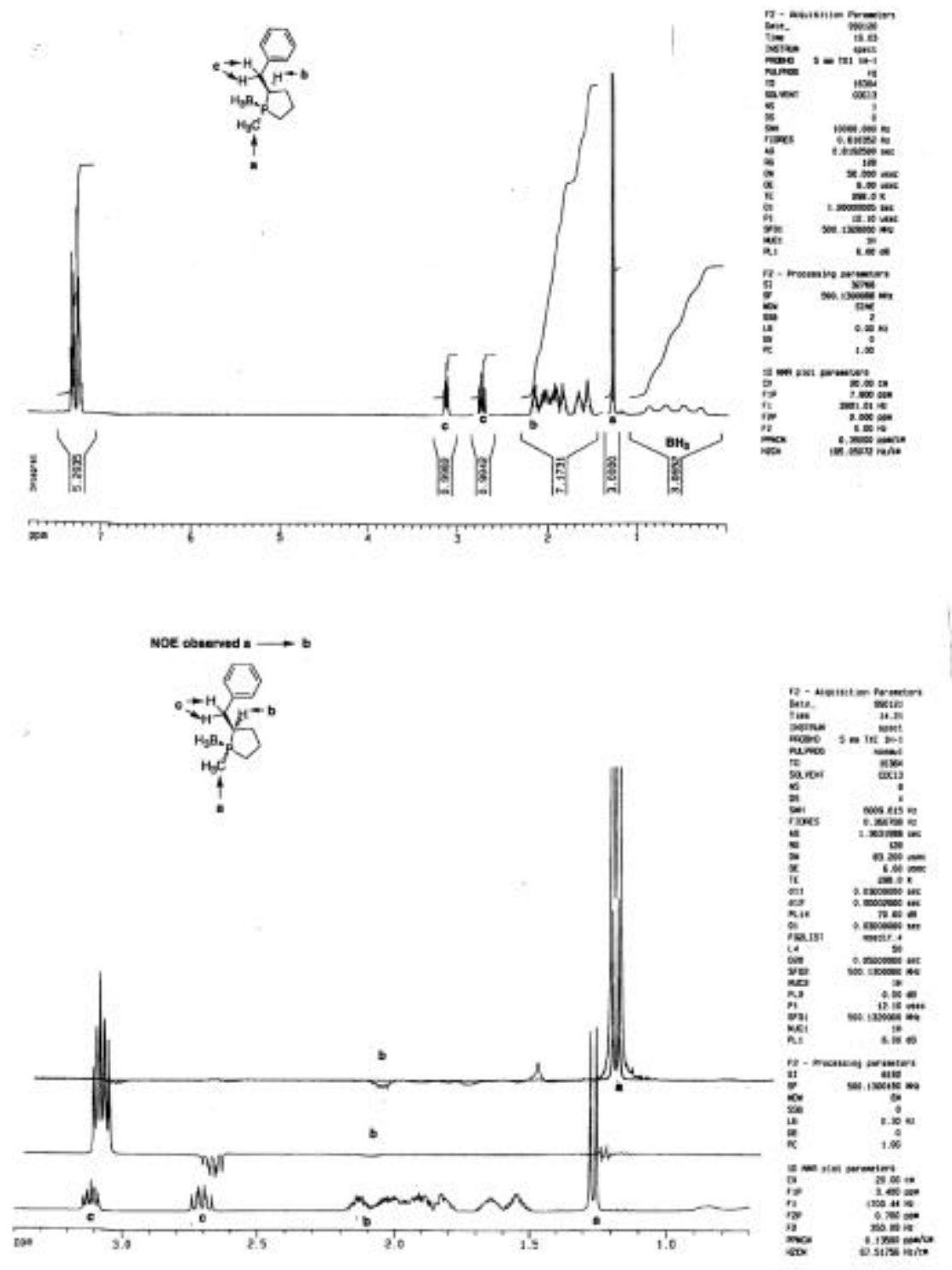

Figure 3S. ${ }^{1} \mathrm{H}$ NMR NOE difference spectra for compound $20\left(500 \mathrm{MHz}, \mathrm{CDCl}_{3}\right)$. 
X-ray Crystal Structure Analysis: Compound $\mathbf{4 8} 43$

\begin{tabular}{|c|c|}
\hline Orpatal data & \\
\hline $\mathrm{C}_{15} \mathrm{H}_{20} \mathrm{O}_{3} \mathrm{~PB}$ & $D_{n}=1.30 \mathrm{Mg} \mathrm{m}^{-3}$ \\
\hline$M_{r}=300.2$ & $\mathrm{X}$-ray radiatios \\
\hline Monoclinic & $\lambda=1.54056 \hat{\AA}$ \\
\hline$P 2_{1}$ & Cell parameters from rellections \\
\hline$a=14.36(5) \hat{A}$ & $0=1-^{\circ}$ \\
\hline$\Delta=6.58(5) \AA$ & $\mu=19.34 \mathrm{~mm}^{-1}$ \\
\hline$c=20.31(5) A$ & $T=298 \mathrm{~K}$ \\
\hline$D=104.3(2)^{6}$ & Plate \\
\hline$V=1859.340(1) \AA^{3}$ & $0.2 \times 0.2 \times 0.2 \mathrm{~mm}$ \\
\hline$z=4$ & Colouriess \\
\hline$D_{n}=1.07 \mathrm{Mg} \mathrm{m} \mathrm{m}^{-2}$ & Crystel source: Local laberatory \\
\hline
\end{tabular}

Dats colbectiont Earaf Nonius CAD-4. Cell refaement: CAD-4. Dats reductiont $\mathrm{maXuas}$. Program(a) used to salve atrocture maXus. Program(a) wed to refine structure: maXua. Molecalar graphies: maXus. Software used to prepare material for publicatious maXus.

\begin{tabular}{|c|c|}
\hline $\begin{array}{l}\text { Abarptioa correction: } \\
\text { none }\end{array}$ & $\begin{array}{l}\theta_{\text {max }}=48.99^{\circ} \\
A=-15 \rightarrow 15\end{array}$ \\
\hline 5561 mesaured reflections & $k=-6 \rightarrow 6$ \\
\hline 4737 Independent refiections & $t=-22 \rightarrow 22$ \\
\hline \multicolumn{2}{|l|}{4430 observed reflections } \\
\hline \multicolumn{2}{|l|}{ Refinement } \\
\hline Refinernent os $F$ & $(\Delta / \sigma)_{\max }=2.7098$ \\
\hline$R=0.096$ & $\Delta P_{\max }=0,48 \in A^{-3}$ \\
\hline$R=0.183$ & $\Delta p_{\text {nia }}=-0.44 \cdot \AA^{-3}$ \\
\hline$S=4.413$ & Extinction correction: Nose \\
\hline 4430 reflections & Atomic scatterias factors from \\
\hline 365 parameters & D. WhasmaierleA. Kirfel, Aeta \\
\hline Only coordinates of $\mathrm{H}$ atoms refised & Cryst. $1995,451,416-451$ \\
\hline Count statistica & \\
\hline
\end{tabular}




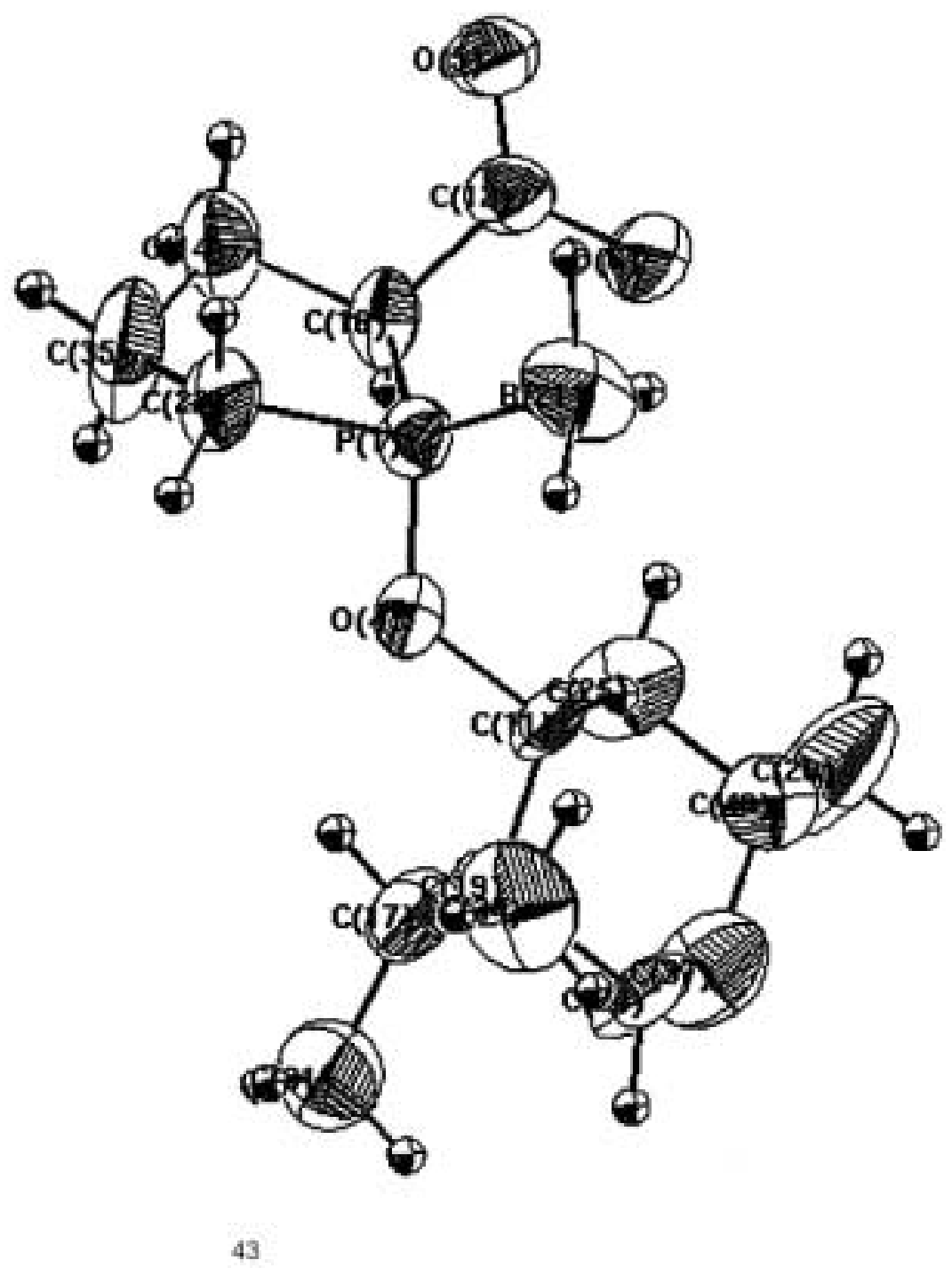

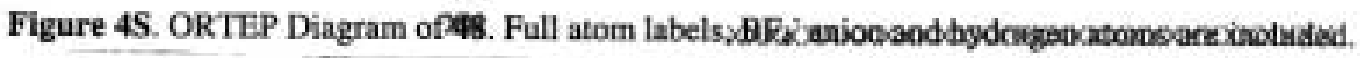


Table 1S. Fractional atomic coondinates and equivalent isotropic displacement porameters $\left(\grave{A}^{2}\right)$ for maxus (compound 43 )

\begin{tabular}{|c|c|c|c|c|}
\hline \multicolumn{5}{|c|}{$U_{\text {eaq }}=(1 / 3) \Sigma_{i} \Sigma_{j} U_{i j} a_{i}^{*} a_{j}^{*} a_{i} \cdot a_{j}}$. \\
\hline & $x$ & $y$ & $x$ & $U_{\text {eq }}$ \\
\hline P1 & $0.01278(6)$ & 0.18700 & $-0.33461(4)$ & $0.0469(6)$ \\
\hline $\mathrm{P}_{2}$ & $-0.48595(6)$ & $-0.0248(2)$ & $-0.39482(4)$ & $0.0490(6)$ \\
\hline O3 & $-0.4753(1)$ & $-0.1507(4)$ & $-0.26336(9)$ & $0.057(2)$ \\
\hline O4 & $0.0603(1)$ & $0.2755(5)$ & $-0.25125(9)$ & $0.056(2)$ \\
\hline 05 & $-0.1423(1)$ & $0.3996(6)$ & $-0.4994(1)$ & 0.077 (2) \\
\hline $\mathrm{O} 6$ & $-0.1988(2)$ & $0.3821(6)$ & $-0.4086(1)$ & $0.084(2)$ \\
\hline $\mathrm{CP}$ & $-0.5364(2)$ & $-0.2434(6)$ & $-0.1699(2)$ & $0.059(2)$ \\
\hline $\mathrm{ClO}$ & $-0.4944(2)$ & $-0.0745(6)$ & $-0.2063(2)$ & $0.055(2)$ \\
\hline $\mathrm{C} 11$ & $-0.0180(2)$ & $0.2495(7)$ & $-0.2113(2)$ & $0.058(2)$ \\
\hline $\mathrm{C} 12$ & $-0.1267(2)$ & $0.3685(7)$ & $-0.4401(2)$ & $0.053(3)$ \\
\hline $\mathrm{Cl3}$ & $-0.5203(2)$ & $-0.2231(8)$ & $=0.3968(1)$ & $0.062(3)$ \\
\hline $\mathrm{Cl4}$ & $0.0466(3)$ & $0.4637(8)$ & $-0.4306(2)$ & $0.077(3)$ \\
\hline $\mathrm{Cls}$ & $-0.6182(2)$ & $-0.2035(7)$ & $-0.4440(2)$ & $0.057(3)$ \\
\hline $\mathrm{C} 16$ & $-0.0291(2)$ & $0.4002(6)$ & $-0.3527(2)$ & $0.048(2)$ \\
\hline $\mathrm{Cl7}$ & $0.1331(3)$ & $0.1208(7)$ & $-0.1253(2)$ & $0.081(3)$ \\
\hline $\mathrm{Cl} 8$ & $-0.4051(2)$ & $0.0223(7)$ & $-0.1601(2)$ & $0.068(3)$ \\
\hline C19 & $0.0474(2)$ & $0.2766(6)$ & $-0.1412(2)$ & $=0.061(2)$ \\
\hline $\mathrm{C} 20$ & $-0.6266(3)$ & $-0.3369(7)$ & $-0.2159(2)$ & $0.068(3)$ \\
\hline B21 & $-0.0573(4)$ & $-0.0473(9)$ & $-0.3445(2)$ & $0.079(4)$ \\
\hline $\mathrm{C} 22$ & $0.1270(2)$ & $0.1690(8)$ & $-0.3568(2)$ & $0.070(3)$ \\
\hline $\mathrm{C} 23$ & $-0.4148(3)$ & $0.0844(7)$ & $-0.0918(2)$ & $0.076(3)$ \\
\hline $\mathrm{C} 24$ & $-0.0129(3)$ & $0.2708(8)$ & $-0.0875(2)$ & $0.077(3)$ \\
\hline C2S & $-0.1001(3)$ & $0.4072(7)$ & $-0.2285(2)$ & $0.077(3)$ \\
\hline $\mathrm{C} 26$ & $-0.1576(3)$ & $0.4073(7)$ & $-0.1732(2)$ & $0.085(3)$ \\
\hline $\mathrm{C} 27$ & $-0.7096(2)$ & $-0.1850(8)$ & $-0.2344(2)$ & $0.107(3)$ \\
\hline C28 & $-0.3461(2)$ & $-0.180(1)$ & $-0.3842(2)$ & $0.089(3)$ \\
\hline $\mathrm{C} 29$ & $-0.4512(3)$ & $-0.0860(9)$ & $-0.0577(2)$ & $0.100(4)$ \\
\hline C30 & $-0.4392(3)$ & $-0.2337(9)$ & $-0.4336(2)$ & $0.094(4)$ \\
\hline $\mathrm{C} 31$ & $0.2083(3)$ & $0.1736(9)$ & $-0.0607(2)$ & $0.099(4)$ \\
\hline $\mathrm{Caz}$ & $-0.3642(2)$ & $0.0019(9)$ & $-0.3455(2)$ & $0.082(3)$ \\
\hline C33 & $-0.5450(3)$ & $-0.1755(8)$ & $-0.1013(2)$ & $0.079(3)$ \\
\hline C34 & $0.1014(3)$ & $-0.0936(8)$ & $-0.1206(2)$ & $0.097(3)$ \\
\hline C35 & $0.1388(3)$ & $0.3700(9)$ & $-0.3869(2)$ & $0.110(4)$ \\
\hline C36 & $-0.6585(3)$ & $-0.5267(8)$ & $-0.1857(2)$ & $0.113(4)$ \\
\hline $\mathrm{C} 37$ & $-0.0944(3)$ & $0.4287(8)$ & $-0.1033(2)$ & $0.100(4)$ \\
\hline Cas & $-0.3214(3)$ & $0.1762(9)$ & $-0.0488(2)$ & $0.115(4)$ \\
\hline
\end{tabular}




\begin{tabular}{|c|c|c|c|c|}
\hline H9 & -0.19104 & -0.35308 & -0.16076 & 0.05000 \\
\hline $\mathrm{H} 10$ & -0.54260 & 0.02945 & -0.21760 & 0.05000 \\
\hline H11 & -0.04512 & 0.11533 & -0.21623 & 0.05000 \\
\hline H13 & -0.52081 & -0.35019 & -0.37365 & 0.05000 \\
\hline $\mathrm{H} 14 \mathrm{~A}$ & 0.03472 & 0.36704 & -0.47214 & 0.05000 \\
\hline $\mathrm{H} 14 \mathrm{~B}$ & 0.05002 & 0.58574 & -0.44064 & 0.05000 \\
\hline H16 & -0.03536 & 0.51369 & -0.36445 & 0.05000 \\
\hline H17 & 0.16252 & 0.12833 & -0.16285 & 0.05000 \\
\hline H18A & -0.35175 & -0.06976 & -0.15423 & 0.05000 \\
\hline H18B & -0.39185 & 0.14234 & -0.18313 & 0.05000 \\
\hline H19 & 0.07595 & 0.40891 & -0.14007 & 0.05000 \\
\hline $\mathrm{H} 2 \mathrm{O}$ & -0.61141 & -0.38390 & -0.25684 & 0.05000 \\
\hline $\mathrm{H} 22 \mathrm{~A}$ & 0.17888 & 0.13855 & -0.31818 & 0.05000 \\
\hline H22B & 0.12588 & 0.07075 & -0.39188 & 0.05000 \\
\hline Н 23 & -0.46453 & 0.18520 & -0.09745 & 0.05000 \\
\hline $\mathrm{H} 24 \mathrm{~A}$ & -0.04582 & 0.14272 & -0.09095 & 0.05000 \\
\hline $\mathrm{H} 24 \mathrm{~B}$ & 0.02578 & 0.28852 & -0.04215 & 0.05000 \\
\hline $\mathrm{H} 25 \mathrm{~A}$ & -0.07048 & 0.53892 & -0.22445 & 0.05000 \\
\hline $\mathrm{H} 25 \mathrm{~B}$ & -0.14058 & 0.39102 & -0.27345 & 0.05000 \\
\hline H26 & -0.18527 & 0.27382 & -0.17608 & 0.05000 \\
\hline H27A & -0.68758 & -0.07358 & -0.25571 & 0.05000 \\
\hline $\mathrm{H} 27 \mathrm{~B}$ & -0.76508 & -0.24598 & -0.26501 & 0.05000 \\
\hline $\mathrm{H} 27 \mathrm{C}$ & -0.72618 & -0.14428 & -0.19371 & 0.05000 \\
\hline $\mathrm{H} 28 \mathrm{~A}$ & -0.33421 & -0.29383 & -0.35394 & 0.05000 \\
\hline $\mathrm{H} 28 \mathrm{~B}$ & -0.29281 & -0.16233 & -0.40444 & 0.05000 \\
\hline H29A & -0.40244 & -0.18915 & -0.04973 & 0.05000 \\
\hline H29B & -0.46204 & -0.04205 & -0.01513 & 0.05000 \\
\hline $\mathrm{H} 30 \mathrm{~A}$ & -0.43192 & -0.35788 & -0.45659 & 0.05000 \\
\hline H30B & -0.44962 & -0.12318 & -0.46549 & 0.05000 \\
\hline H31А & 0.23026 & 0.31088 & -0.06221 & 0.05000 \\
\hline
\end{tabular}




$\begin{array}{lrrrl}\text { Table 1S (continued) } & & & \\ \text { H32A } & -0.35813 & 0.11969 & -0.37198 & 0.05000 \\ \text { H32B } & -0.31563 & 0.00519 & -0.30348 & 0.05000 \\ \text { H33A } & -0.59422 & -0.07335 & -0.10835 & 0.05000 \\ \text { H33B } & -0.56472 & -0.29105 & -0.07915 & 0.05000 \\ \text { H34A } & 0.05423 & -0.13061 & -0.16110 & 0.05000 \\ \text { H34B } & 0.15603 & -0.18211 & -0.11480 & 0.05000 \\ \text { H34C } & 0.07423 & -0.10601 & -0.08210 & 0.05000 \\ \text { H35A } & 0.18807 & 0.36557 & -0.41145 & 0.05000 \\ \text { H35B } & 0.15747 & 0.46217 & -0.34935 & 0.05000 \\ \text { H36A } & -0.60637 & -0.62240 & -0.17547 & 0.05000 \\ \text { H36B } & -0.67457 & -0.48680 & -0.14437 & 0.05000 \\ \text { H36C } & -0.71337 & -0.58850 & -0.21567 & 0.05000 \\ \text { H37A } & -0.13048 & 0.43758 & -0.06936 & 0.05000 \\ \text { H37B } & -0.06258 & 0.55578 & -0.10586 & 0.05000 \\ \text { H38A } & -0.30426 & 0.28838 & -0.07368 & 0.05000 \\ \text { H38B } & -0.33006 & 0.22368 & -0.00598 & 0.05000 \\ \text { H38C } & -0.27106 & 0.07638 & -0.04098 & 0.05000 \\ \text { H40A } & -0.28027 & 0.53827 & -0.23373 & 0.05000 \\ \text { H40B } & -0.27257 & 0.57737 & -0.15653 & 0.05000 \\ \text { H40C } & -0.20327 & 0.69037 & -0.19303 & 0.05000 \\ \text { H21A } & -0.07512 & -0.09914 & -0.39021 & 0.019(7) \\ \text { H21B } & -0.02092 & -0.14994 & -0.31571 & 0.042(9) \\ \text { H21C } & -0.11492 & -0.01994 & -0.33021 & 0.09(2) \\ \text { H8 } & -0.72457 & -0.33578 & -0.43254 & 0.12(1) \\ \text { H6 } & -0.23324 & 0.25605 & -0.41768 & 0.16(1) \\ & & & & \end{array}$




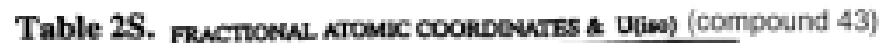

\begin{tabular}{|c|c|c|c|c|}
\hline A10en & 2/n & $y_{0}^{6}$ & Dto & U(as) \\
\hline$\overline{\text { (i) }}$ & $0.01273(6)$ & 218750 & $.0 .35461(4)$ & $006646)$ \\
\hline$P(2)$ & $.43595(6)$ & $-2004(2)$ & $-0.33482(4)$ & a.0eso(s) \\
\hline o(3) & $-0.753(1)$ & $-0.1500(4)$ & $-0.2633(99)$ & $0.057(2)$ \\
\hline$\alpha(4)$ & $0.0403(1)$ & o.77ss(5) & $-0.26123(9)$ & $0.056(2)$ \\
\hline o(s) & $-0.1423(1)$ & 0334660 & $-0.4994(1)$ & $0.07 \pi(2)$ \\
\hline Q(5) & $-0.1584(2)$ & $0.321(6)$ & $-0.4006(1)$ & $0.014(2)$ \\
\hline orn & $-0.6337(1)$ & $-0.1606(5)$ & .0.sovech & 0.06727 \\
\hline O(B) & $-0.6 \sin 2(2)$ & $0.2208(5)$ & $-0.4121(1)$ & $0.074(2)$ \\
\hline$\left.C_{9}\right)$ & $.0 .53542)$ & $-0.2434(6)$ & $-0.1609(2)$ & $0.059(2)$ \\
\hline$c(10)$ & $0.9442)$ & $-0.0146(6)$ & $-0.2063(2)$ & $\operatorname{aoss}(2)$ \\
\hline criil & $-0.0 \operatorname{ls} 0(2)$ & $0.2698(7)$ & $-0.2113(2)$ & $0.058(2)$ \\
\hline $\mathrm{C}(12)$ & $-0.1267(7)$ & $0.3643(7)$ & $-0.4401(2)$ & $0.053(3)$ \\
\hline$C(13)$ & $-0.5203(2)$ & $-0.231(b)$ & a.seces (1) & $0.062(3)$ \\
\hline $\mathrm{C}(14)$ & $0.0406(3)$ & $0.443 \times(8)$ & $-0.4306(2)$ & $0.0723)$ \\
\hline$\alpha(5)$ & -0.6132027 & $-0.2036(7)$ & $-0.4440(2)$ & $0.057(3)$ \\
\hline Q16) & $-0.001(2)$ & $0.4002(8)$ & $-0.3 \sin (2)$ & $0.043(2)$ \\
\hline cin & $0.1331(3)$ & Q.1208(7) & $-0.1253(2)$ & acsicis \\
\hline c(18) & $0 . \cos 1(2)$ & $0.0223(n)$ & $-0.1601(2)$ & $0.06 t(3)$ \\
\hline ca9s & $0.0474(2)$ & $0.2766(6)$ & -0.1612a) & $0.061(2)$ \\
\hline $\mathrm{Cr}(\mathrm{a})$ & $-0.6268(3)$ & $-0.3360(7)$ & $-0.2159(2)$ & 0.06803 \\
\hline B(2t) & $-0.0573(0)$ & -0.04739 & $-0.3446(2)$ & $0.079(4)$ \\
\hline $\mathrm{C}(22)$ & 0.12700 ) & $0.1650 \times 1)$ & $-0.3568(2)$ & $0.000(3)$ \\
\hline$C(23)$ & -0.414803 & 0.084477 & $-0.0918(2)$ & $0.076(3)$ \\
\hline$C(24)$ & $-0.0125(3)$ & $0.2308(1)$ & $0.0575(2)$ & $0.07 /(3)$ \\
\hline$c(29)$ & $2.1001(3)$ & 0.4งก:7) & $-0.2285(2)$ & $0.07(3)$ \\
\hline C(26) & $0.1576(3)$ & $0.4073(7)$ & $-0.1732(2)$ & a.cesp) \\
\hline $\mathrm{c} 27)$ & $-0.70962)$ & - a.1800(8) & $-0.2344(2)$ & $0.1030)$ \\
\hline$c(28)$ & $0.3461(2)$ & $-0.160(1)$ & $-0.3442(2)$ & $\operatorname{acs} 301$ \\
\hline C(29) & $0.5512(3)$ & acssog) & $-0.0 s 77(2)$ & $0.100(4)$ \\
\hline $\mathrm{C}(30)$ & $-0.692(3)$ & - ..23379 & $-0.4336(2)$ & $0.094(4)$ \\
\hline $\mathrm{C}(31)$ & $0.2563(3)$ & $0.1736 \mathrm{n}$ & $-0.0607(2)$ & $0.009(4)$ \\
\hline$C(32)$ & $-0.3642(2)$ & $0.0019(9)$ & $-0.3498(2)$ & a.osics) \\
\hline$C(33)$ & $-0.5490(3)$ & $-0.1758(8)$ & $-0.1013 \times 27$ & $0.079(3)$ \\
\hline $\mathrm{CO4})$ & $0.1014(3)$ & $-0.003 \times(8)$ & $-0.1206(2)$ & $0.057(3)$ \\
\hline C(35) & Q.1338(p) & $0.3700(9)$ & $=0.3869(2)$ & $0.110(4)$ \\
\hline co6) & $-0.5835(3)$ & $.05262(8)$ & $-0.1357(2)$ & $0.113(4)$ \\
\hline clati & $-0.00443)$ & $0.4287(8)$ & $-0.1039(2)$ & $0.100(4)$ \\
\hline c(38) & $-0.32143)$ & $0.1762(9)$ & $-0.0431(2)$ & $0.115(4)$ \\
\hline $\mathrm{D}(39)$ & $0.5561(3)$ & $0.2290(8)$ & $-0.3419(2)$ & $0.073(4)$ \\
\hline$C(40)$ & $0.2369(3)$ & $0.566(1)$ & $-0.1909(2)$ & $0.158(5)$ \\
\hline H(O) & 0.09104 & .0 .35308 & -0.16076 & 0.05000 \\
\hline หัฒ & .0 .54260 & 0 cosiss & 0.21760 & 0.05000 \\
\hline $1 \times(1)$ & -0.04512 & 0.11533 & -0.21623 & 0.05000 \\
\hline HX133 & .0 .52081 & -035019 & -0.37365 & 0.05000 \\
\hline $\mathrm{H}(14 \mathrm{~A})$ & 0.0347 & $0.36 \pi 04$ & .0 .7214 & 0.05000 \\
\hline B(14B) & 0.00002 & 0.51574 & 0.44064 & $0.6 \$ 000$ \\
\hline$H(16)$ & 0.03536 & 0.91369 & -0.36445 & 9.09000 \\
\hline $\mathrm{H}[17)$ & 0.16252 & 0.12833 & -0.16215 & 0.05000 \\
\hline
\end{tabular}


Table 2S (continued)

\begin{tabular}{|c|c|c|c|c|}
\hline$\overline{H(18 A)}$ & -0.35175 & -0.06976 & -0.15423 & 0.05000 \\
\hline$H(18 B)$ & -0.39185 & 0.14234 & $=0.18313$ & 0.05000 \\
\hline$H(19)$ & 0.07595 & 0.40891 & -0.14007 & 0.05000 \\
\hline$H(20)$ & -0.61141 & -0.38390 & -0.25684 & 0.05000 \\
\hline$H(22 A)$ & 0.17888 & 0.13855 & -0.31818 & 0.05000 \\
\hline $\mathrm{H}(22 \mathrm{~B})$ & 0.12588 & 0.07075 & -0.39188 & 0.05000 \\
\hline$H(23)$ & -0.46453 & 0.18520 & -0.09745 & 0.05000 \\
\hline$H(24 A)$ & -0.04582 & 0.14272 & -0.09095 & 0.05000 \\
\hline $\mathrm{H}(24 \mathrm{~B})$ & 0.02578 & 0.28852 & -0.04215 & 0.05000 \\
\hline$H(25 A)$ & -0.07048 & 0.53892 & -0.22445 & 0.05000 \\
\hline $\mathrm{H}(25 \mathrm{~B})$ & -0.14058 & 0.39102 & -0.27345 & 0.05000 \\
\hline$H(26)$ & -0.18527 & 0.27382 & -0.17608 & 0.05000 \\
\hline$H(27 \mathrm{~A})$ & -0.68758 & -0.07353 & -0.25571 & 0.05000 \\
\hline $\mathrm{H}(27 \mathrm{~B})$ & -0.76508 & -0.24598 & -0.26501 & 0.05000 \\
\hline$H(27 C)$ & -0.72618 & -0.14423 & -0.19371 & 0.05000 \\
\hline $\mathrm{H}(28 \mathrm{~A})$ & -0.33421 & -0.29383 & -0.35394 & 0.05000 \\
\hline $\mathrm{H}(28 \mathrm{~B})$ & -0.29281 & -0.16233 & -0.40444 & 0.05000 \\
\hline $\mathrm{H}(29 \mathrm{~A})$ & -0.40244 & 0.18915 & -0.04973 & 0.05000 \\
\hline $\mathrm{H}(29 \mathrm{~B})$ & -0.46204 & -0.04205 & -0.01513 & 0.05000 \\
\hline $\mathrm{H}(30 \mathrm{~A})$ & -0.43192 & .0 .35788 & -0.45659 & 0.05000 \\
\hline $\mathrm{H}(303)$ & -0.44962 & 0.12318 & -0.46549 & 0.05000 \\
\hline$H(31 A)$ & 0.23026 & 0.31088 & -0.06221 & 0.05000 \\
\hline$H(313)$ & 0.17986 & 0.15808 & -0.02291 & 0.05000 \\
\hline$H(31 C)$ & 0.26166 & 0.08198 & .0 .05561 & 0.05000 \\
\hline $\mathrm{H}(32 \mathrm{~A})$ & -0.35813 & 0.11969 & $=0.37198$ & 0,05000 \\
\hline $\mathrm{H}(32 \mathrm{~B})$ & -0.31563 & 0.00519 & -0.30348 & 0.05000 \\
\hline $\mathrm{H}(33 \mathrm{~A})$ & -0.59422 & 0.07335 & -0.10835 & 0.05000 \\
\hline $\mathrm{H}(33 \mathrm{~B})$ & -0.36472 & -0.29105 & -0.07915 & 0.05000 \\
\hline$H(34 \Lambda)$ & 0.05123 & -0.13061 & -0.16110 & 0.05000 \\
\hline$H(34 B)$ & 0.15603 & -0.18211 & 0.11480 & 0.05000 \\
\hline $\mathrm{H}(34 \mathrm{C})$ & 0.07423 & -0.10601 & -0.08210 & 0.05000 \\
\hline$H(35 N)$ & 0.18807 & 0.35557 & -0.41145 & 0.05000 \\
\hline$H(35 B)$ & 0.15747 & 0.45217 & -0.34935 & 0.05000 \\
\hline$H(36 A)$ & -0.60637 & .0 .62260 & -0.17547 & 0.05000 \\
\hline $\mathrm{H}(36 \mathrm{~B})$ & -0.67457 & -0.48680 & -0.14437 & 0.05000 \\
\hline $\mathrm{H}(36 \mathrm{C})$ & -0.71337 & 0.58850 & -0.21567 & 0.05000 \\
\hline$H(37 A)$ & -0.13048 & 0.43758 & -0.06936 & 0.05000 \\
\hline$H(37 B)$ & -0.06258 & 0.55578 & -0.10585 & 0.05000 \\
\hline H(38A) & -0.30426 & 0.28838 & -0.07368 & 0.05000 \\
\hline$H(38 B)$ & -0.33006 & 0.22368 & 0.00598 & 0.05000 \\
\hline$H(38 C)$ & -0.27106 & 0.07638 & -0.04098 & 0.05000 \\
\hline $\mathrm{H}(40 \mathrm{~A})$ & -0.28027 & 0.53827 & .023373 & 0.05000 \\
\hline H(40D) & -0.27257 & 0.57737 & 0.15653 & 0.05000 \\
\hline $\mathrm{H}(40 C)$ & -0.20327 & 0.69037 & -0.19303 & 0.05000 \\
\hline$H(2 \mid A)$ & 0.07512 & -0.09914 & -0.39021 & $0.019(7)$ \\
\hline $\mathrm{H}(2 \mid \mathrm{B})$ & -0.02092 & -0.14994 & -0.31571 & $0.042(9)$ \\
\hline$H(2 \mid C)$ & -0.11492 & -0.01994 & -0.33021 & $0.09(2)$ \\
\hline $\mathrm{H}(\mathrm{B})$ & -0.72457 & -0.33578 & -0.43254 & $0.12(1)$ \\
\hline $\mathrm{H}(6)$ & -0.23324 & 0.25605 & -0.41763 & $0.16(1)$ \\
\hline
\end{tabular}




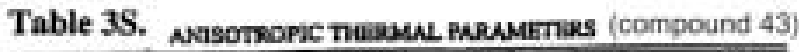

\begin{tabular}{|c|c|c|c|c|c|c|}
\hline om & UII & un & 023 & 012 & DII & 023 \\
\hline$\overline{\text { F1) }}$ & Essis & $050607)$ & $0.0 \mathrm{~s}(\mathrm{~s})(5)$ & $0.005 \times 101$ & $26151(4)$ & $20019 \times 6)$ \\
\hline & $0.52 \times 35 ?$ & a. $0528(n)$ & & & (4) & $\infty 003 \times 16$ \\
\hline 3 & rest & $\cos (2)$ & $044(1)$ & $\cos \theta(1)$ & & $0.005(1)$ \\
\hline & $94(1)$ & & & min & $0.015(1)$ & $0.010(1)$ \\
\hline & & $39(9)$ & $044(1)$ & $0.016(2)$ & $013(1)$ & $-.003(2)$ \\
\hline & 年 & & & $0 . \cos (2)$ & ansil & $-\operatorname{sic}(2)$ \\
\hline & (at) & & & $-\cos (2)$ & i) & $0.00007)$ \\
\hline & 259 & & & $015(2)$ & & $0.006(2)$ \\
\hline & & & & & $0.016(2)$ & $0.000(2)$ \\
\hline & 48 & & & 0.00 & 5 &.,$\infty 1(2)$ \\
\hline & & & & & $\cos (2)$ & $0000(2)$ \\
\hline & 0.06 & & & 0.0 : & $m(2)$ & $00000(2)$ \\
\hline & & & & & $\cos (2)$ & $0.000(3)$ \\
\hline & A & & & $.0005(3)$ & & aces(3) \\
\hline 15 & & & & & & $-\cos (x)$ \\
\hline & & & & 0.0 & & $0.013 a)$ \\
\hline & & & & & & $0002(3)$ \\
\hline & & & & 0.0 & & 00 \\
\hline & & & & & 0 & 0.0 \\
\hline & & & & 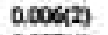 & & \\
\hline & & & & & & $-014(3)$ \\
\hline & & & & 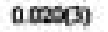 & 2) & $0.004(3)$ \\
\hline 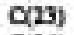 & & & & & & \\
\hline & & & & 0.0 & 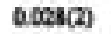 & 001201 \\
\hline & & & & & & 2) \\
\hline & 0.8 & & & & & $0.016(3)$ \\
\hline & & & & . an & & $0.000(4)$ \\
\hline & & & & & & $.007 x$ \\
\hline & & & & $-\infty$ & & \\
\hline & & & & & & \\
\hline & & & & & & \\
\hline & & & & & & \\
\hline$c a$ & & & & & & .00003 \\
\hline$\alpha$ & 0.04 & & & & & 0.03107 \\
\hline$\infty$ & & & & $m$ & & 0.03140 \\
\hline ca & & & & & & \\
\hline$c$ & 0.09 & & & & o. & 0.002019 \\
\hline & & & & & & \\
\hline & & & & & & \\
\hline-1 & $0.100(0)$ & 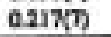 & 19644 & $0.04(4)$ & $0.005(3)$ & ous2 \\
\hline
\end{tabular}

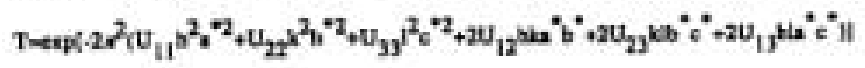


Table 4S. DNTRAMoLncuLAR BOND Leverns or eminad) (compound 43)

\begin{tabular}{|c|c|c|c|}
\hline \multicolumn{4}{|c|}{ 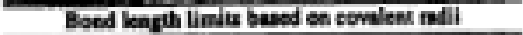 } \\
\hline राम्ब(4) & 1.35703 & स(1)व(6) & $\overline{1.537(4)}$ \\
\hline P(1)-B(2i) & 1.22506 & (1) (1) & $1.803(4)$ \\
\hline$P(2)-0(3)$ & 1.55935 & (2)-व(13) & $1.795(5)$ \\
\hline $\mathrm{P}(2) \mathrm{C}(32)$ & $1.822(4)$ & o(3)-Q1010 & $1.44(4)$ \\
\hline Q(3) $\alpha(12)$ & $1.183(4)$ & ஊ(5-व12) & $1.34 \pi(4)$ \\
\hline$\alpha(T) \alpha(15)$ & $1.212(4)$ & (8)-C(1) & $1.316(4)$ \\
\hline CQj-C(33) & $1.497(5)$ & Q13)-Q(5) & $1.5000(5)$ \\
\hline q(14)-cas) & $1.483(6)$ & Q(17)-C(34) & L693(n) \\
\hline Q(1)-C(23) & $1.485(5)$ & c(22)-c(35) & LAS(3) \\
\hline C23)-C29) & 1.47967 & C201) व(3) & $1.45(6)$ \\
\hline$\alpha(2)-\alpha(3)$ & $1,438(5)$ & & \\
\hline
\end{tabular}

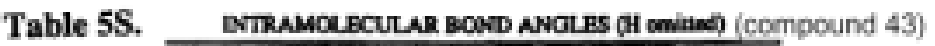

\begin{tabular}{|c|c|c|c|}
\hline \multicolumn{4}{|c|}{ 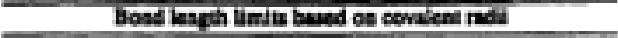 } \\
\hline (0)-7(1)-C(16) & $\sin 2(2)$ & $O(0)-\pi(1)-\Delta(21)$ & $1132(2)$ \\
\hline$O(4)-\mathrm{R}(1)-\mathrm{C} 22)$ & $103.3(2)$ & $\mathrm{C}(16)-P(1)-\mathrm{B}(21)$ & 119302 \\
\hline Q(16-r(1)-can) & $93.6(2)$ & $B(21)-P(1)-O(23)$ & 115.200 \\
\hline $0(3)-(2)-(203)$ & $99.9(z)$ & O(3)-P(2)-C(12) & $10822(2)$ \\
\hline$\alpha_{1}(3)-F(2)-C(2)$ & $95.3(2)$ & $P(2)-0(1)-C(10)$ & $125.03)$ \\
\hline $0(3)-\mathrm{C}(12)-\mathrm{O}(6)$ & $121.2(3)$ & $P(2)-C(13)-C(15)$ & $116.1(4)$ \\
\hline O(7)-C(15)-O(5) & 122.901 & $\alpha 7)-(15)-\alpha(3)$ & $124.9(3)$ \\
\hline$\alpha(8)-C(15)-(13)$ & $112.2(3)$ & $P(1)-C(23)-C(35)$ & $104.3(4)$ \\
\hline Q(18)-C(25)-C(29) & 110.210 & $P(2)-(3)-(x)$ & $106.5(4)$ \\
\hline$(16)-c(35)-c(2 x)$ & $1113 / 9)$ & & \\
\hline
\end{tabular}

Table 6S.

ITRAMCLECLLAR TORSION ANALAS (H emiand) (Compound 43)

\begin{tabular}{|c|c|c|c|}
\hline \multicolumn{4}{|c|}{ Bond hagth tianiu basod en conkent nadi } \\
\hline $\mathrm{D}(4)-\mathrm{F}(1)-(22)-\mathrm{C}(35)$ & - सब(ग) & Q(6)-R(1)C(2n)-C(5) & 20003 \\
\hline B(21)-W(1)-C(22)-C(35) & $144.4(9)$ & C(13)-P(2)-o(3)-C(10) & $-148.3(3)$ \\
\hline O(B)-(N2)-C(13)-C(15) & $116.43)$ & C(32)-P(a)-C(3)-C(10) & $113.4(3)$ \\
\hline o(3)-P2)-c(3n-C(21) & n.t(4) & C(32)-P(2)-C(13)-C(15) & $-136.6(4)$ \\
\hline$C(13)-P(2)-C(32)-C(2)$ & $-(4 . \alpha(4)$ & $P(2)-\alpha i 3)-(15)-07)$ & $116.0(9)$ \\
\hline$P_{(2)-C(13)-c(15)-0(1)}$ & $-660(4)$ & F(1)-cas-cas)-C(14) & +39.9(4) \\
\hline
\end{tabular}




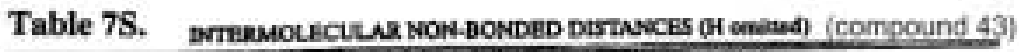

\begin{tabular}{|c|c|c|c|c|c|c|c|c|c|c|}
\hline & & & & & & & & & & \\
\hline Aitan(1)-Nisen(2) & dim & e.a. & as & ne & $\mathbf{n}$ & so & Te & $2(2)$ & & s(2) \\
\hline o(shows & 2685 & 0.003 & 2 & 1 & -1 & $\sigma$ & -1 & -03127 & 0.27912 & .058790 \\
\hline$(5)(9 \mid 4)$ & $34: 4$ & 0.005 & 2 & I & 0 & +1 & -1 & -0.04662 & -0.06626 & .056036 \\
\hline O(5) C(IS) & 3.358 & acos & 2 & 1 & -1 & 0 & +1 & -0.3472 & 0.29536 & -0.59946 \\
\hline Oisforn & 2.637 & 0.003 & 2 & 1 & -1 & 0 & -1 & -036630 & 0.31938 & -0.40502 \\
\hline $0(6)$ Cis & 3507 & 0.004 & $\boldsymbol{2}$ & 1 & -1 & 0 & -1 & -0.3817 & 0.29534 & -0.58546 \\
\hline ortaiz) & 3360 & 0,004 & 2 & 1 & -1 & -1 & -1 & $-0,57800$ & -0.13166 & ossans \\
\hline oricros & 3.496 & 0,006 & 2 & 1 & 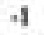 & 0 & -1 & 0.56078 & 0.26632 & -0.5644 \\
\hline$c(12) C(15)$ & 3363 & $a 00 s$ & 2 & $t$ & +1 & 0 & -1 & 0.38179 & 0.29636 & 055595 \\
\hline Cris) B(99) & 3.347 & 0000 & 1 & 1 & 0 & -1 & $\theta$ & 0.55607 & -amias & 0.34151 \\
\hline C(16)B(21) & 3.311 & 0,00 & 1 & I & 0 & 1 & $\theta$ & -0.05732 & 0.95266 & 234461 \\
\hline $\mathrm{C}(1 \mathrm{~s}) \mathrm{Cl}\left({ }_{0}\right)$ & 3.997 & 0.000 & $\mathrm{t}$ & $t$ & 0 & -1 & $\theta$ & -023647 & .043443 & 2.19083 \\
\hline c(22) & 2.785 & 0.006 & $t$ & I & 1 & 0 & 0 & 0.25042 & -0.18798 & -023441 \\
\hline$O(S)-O(B)$ & 2.615 & 0.003 & 2 & $t$ & -1 & 0 & $=1$ & -0.31273 & 027912 & 20.53790 \\
\hline $0(5) C(14)$ & 2.414 & $0.00 s$ & 2 & 1 & 0 & -1 & -1 & 0.04662 & -0.05626 & 405636 \\
\hline o(5)-C(15) & 3.358 & 0.064 & 2 & 1 & -1 & D & -1 & -0.33179 & 0.25636 & -0.55596 \\
\hline $0(6), 0(7)$ & 2637 & 0.003 & 2 & 1 & -1 & 0 & +1 & -0.36630 & 0.31938 & -0.43500 \\
\hline o(6)c(15) & 3507 & 0.004 & 2 & $\mathbf{i}$ & -1 & 0 & -1 & -03317 & 0.29636 & .0 .55596 \\
\hline o(7)c(12) & 3350 & Q.004 & $z$ & i & -1 & -1 & -1 & -0.87390 & 0.13566 & -0.59946 \\
\hline ormeras & 3.456 & 0.006 & 2 & i & -1 & $\mathbf{0}$ & -1 & $-0 \sin \theta$ & 0.26632 & .056641 \\
\hline$C(12)-(15)$ & 3.363 & a.cos & 2 & 1 & -1 & 0 & -1 & -0.38179 & 0.29635 & -0.55596 \\
\hline (1) 13$)-B(99)$ & 3.347 & 0.007 & 1 & 1 & 0 & -1 & 0 & $-0,55607$ & 077103 & $0.3415 !$ \\
\hline C(16)-B(a1) & 3.111 & 0.003 & 1 & 1 & 0 & 1 & 0 & -0.06732 & 0.95366 & -0.34461 \\
\hline C(t) $-C(40)$ & 3.907 & 0.00 & 1 & $t$ & 0 & -1 & 0 & -0.23587 & .0 .43443 & -0.19053 \\
\hline $\mathrm{C}(22)-\mathrm{C}(27)$ & 3.785 & 0.006 & 1 & 1 & 1 & 0 & 0 & 0.20042 & -0.18798 & -0.29441 \\
\hline
\end{tabular}

C(22)-C(27)

$3.7 \mathrm{sin} 0.006$

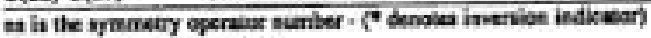

sp it be lesict poiat azmber

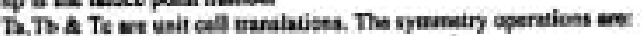

$\mathbf{t}+\mathbf{X}_{1}+\mathbf{Y}_{t}+\mathbf{Z}$

Z $-X, I n+Y, Z$ 


\section{Crystal data}

Empirical formula

Formula weight

Temperature

Wavelength

Crystal system

Space group

Unit cell dimensions

Volume

$\mathrm{Z}$

Density (calculated)

Absorption coefficient

$\mathrm{F}(000)$

Crystal size

Theta range for data collection

Index ranges

Reflections collected

Independent reflections

Completeness to theta $=28.31^{\circ}$

Absorption correction

Max. and min. transmission

Refinement method

Data / restraints / parameters

Goodness-of-fit on $\mathrm{F}^{2}$

Final $\mathrm{R}$ indices [I $>2 \operatorname{sigma}(\mathrm{I})]$

$\mathrm{R}$ indices (all data)

Absolute structure parameter

Extinction coefficient

Largest diff. peak and hole

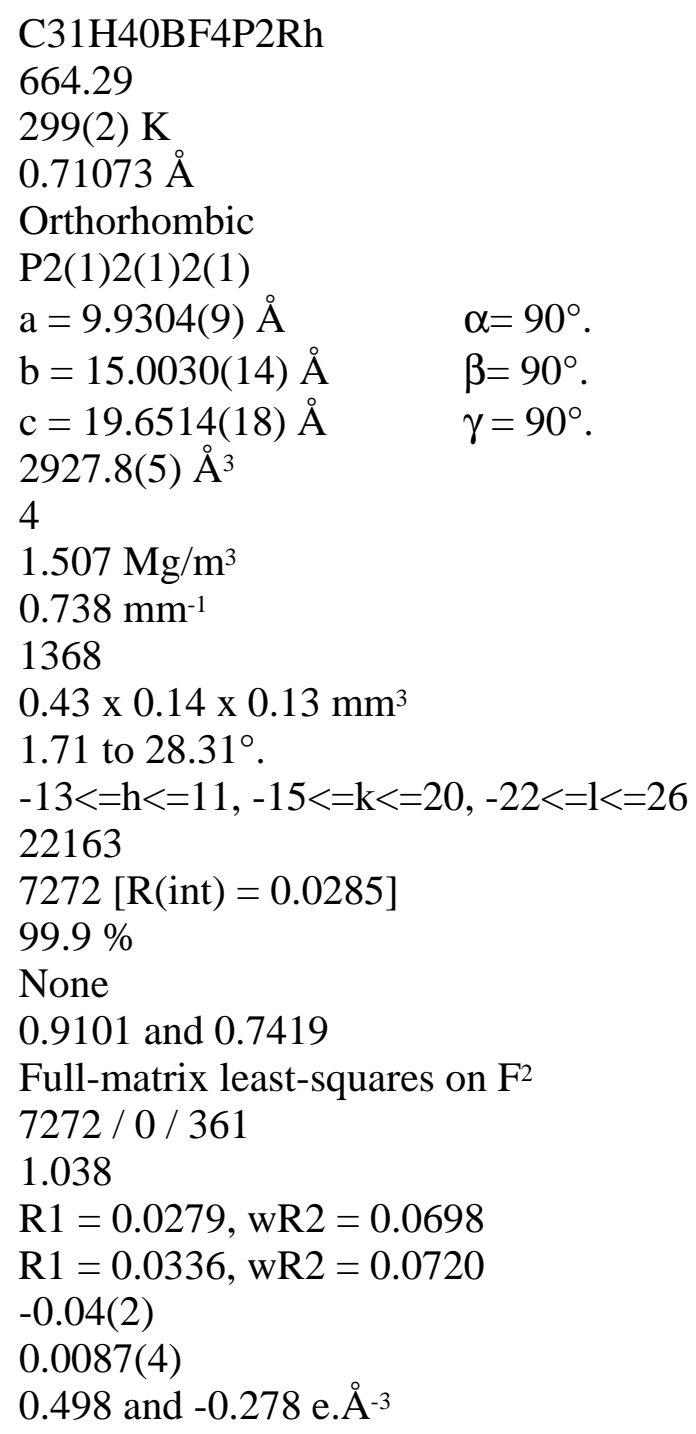




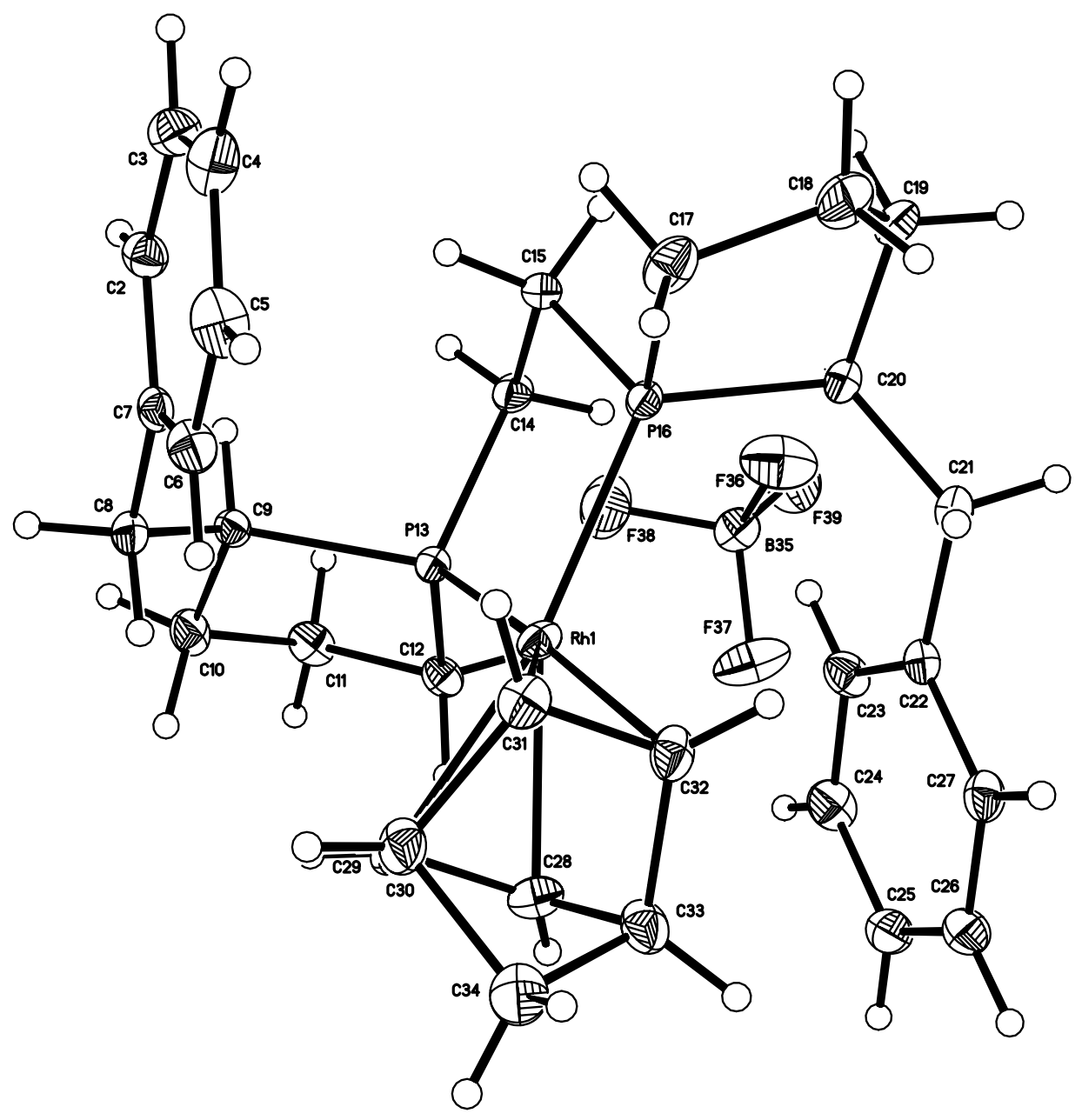

Figure 5S. ORTEP diagram of compound 36. Full atom labels, $\mathrm{BF}_{4}{ }^{-}$counterion and hydrogens are included. 
Table 8S. Atomic coordinates $\left(\mathrm{x} 10^{4}\right)$ and equivalent isotropic displacement parameters $\left(\AA^{2} \mathrm{x} 10^{3}\right)$. $\mathrm{U}(\mathrm{eq})$ is defined as one third of the trace of the orthogonalized $\mathrm{Uij}^{\mathrm{ij}}$ tensor (compound 36).

\begin{tabular}{|c|c|c|c|c|}
\hline & $\mathrm{x}$ & $\mathrm{y}$ & $\mathrm{z}$ & $\mathrm{U}(\mathrm{eq})$ \\
\hline $\mathrm{Rh}(1)$ & $9159(1)$ & $5005(1)$ & $8773(1)$ & $41(1)$ \\
\hline $\mathrm{P}(15)$ & $9813(1)$ & $5877(1)$ & $9680(1)$ & $32(1)$ \\
\hline$C(16)$ & $8735(3)$ & $6764(2)$ & $10005(1)$ & $46(1)$ \\
\hline $\mathrm{C}(18)$ & $9158(3)$ & $5865(2)$ & $11013(1)$ & $58(1)$ \\
\hline$C(19)$ & $10153(3)$ & $5373(2)$ & $10535(1)$ & $38(1)$ \\
\hline$C(20)$ & 10099(3) & $4368(2)$ & $10598(1)$ & $45(1)$ \\
\hline $\mathrm{C}(17)$ & $9020(3)$ & $6813(2)$ & $10767(1)$ & $55(1)$ \\
\hline $\mathrm{P}(12)$ & $11256(1)$ & $5324(1)$ & $8378(1)$ & $36(1)$ \\
\hline $\mathrm{C}(27)$ & $6930(3)$ & $4966(2)$ & $8777(2)$ & $58(1)$ \\
\hline $\mathrm{F}(36)$ & $5668(3)$ & $5037(2)$ & 763(1) & $108(1)$ \\
\hline $\mathrm{F}(38)$ & $5823(3)$ & $6433(2)$ & $1165(2)$ & $116(1)$ \\
\hline $\mathrm{C}(8)$ & $11448(3)$ & $6201(2)$ & $7717(1)$ & $44(1)$ \\
\hline $\mathrm{F}(35)$ & $5388(3)$ & $6174(2)$ & $92(2)$ & 134(1) \\
\hline $\mathrm{C}(31)$ & $8270(3)$ & $4340(2)$ & 7881(1) & $52(1)$ \\
\hline $\mathrm{F}(37)$ & $3803(2)$ & $5897(2)$ & $835(2)$ & 113(1) \\
\hline $\mathrm{C}(13)$ & $12288(2)$ & $5784(2)$ & $9072(1)$ & $45(1)$ \\
\hline $\mathrm{C}(14)$ & $11420(3)$ & $6422(2)$ & $9477(1)$ & $45(1)$ \\
\hline$C(10)$ & $12862(3)$ & $4945(3)$ & $7298(2)$ & $74(1)$ \\
\hline$C(6)$ & 8949(3) & $6570(2)$ & $7448(1)$ & $42(1)$ \\
\hline $\mathrm{C}(7)$ & 10291(3) & $6247(2)$ & 7193(1) & $47(1)$ \\
\hline $\mathrm{C}(21)$ & $11162(3)$ & $3868(2)$ & $10214(1)$ & $42(1)$ \\
\hline $\mathrm{C}(9)$ & 12791(3) & $5950(2)$ & $7375(2)$ & $62(1)$ \\
\hline $\mathrm{C}(4)$ & $6545(3)$ & $6680(2)$ & $7270(2)$ & $59(1)$ \\
\hline $\mathrm{C}(22)$ & 10810(3) & $3254(2)$ & $9721(2)$ & $56(1)$ \\
\hline$C(26)$ & $12527(3)$ & $4012(2)$ & $10339(2)$ & $49(1)$ \\
\hline $\mathrm{C}(5)$ & 7786(3) & $6347(2)$ & 7083(1) & $51(1)$ \\
\hline C(29) & $7526(3)$ & $3480(2)$ & $8805(2)$ & $60(1)$ \\
\hline $\mathrm{C}(1)$ & $8797(3)$ & $7142(2)$ & $8002(1)$ & $54(1)$ \\
\hline$C(30)$ & $8722(3)$ & $3702(2)$ & $8326(2)$ & $53(1)$ \\
\hline $\mathrm{C}(32)$ & $6793(3)$ & $4526(2)$ & $8080(2)$ & $61(1)$ \\
\hline $\mathrm{C}(11)$ & $12318(4)$ & $4499(2)$ & $7948(2)$ & $64(1)$ \\
\hline $\mathrm{C}(28)$ & $7368(3)$ & $4331(2)$ & $9213(2)$ & $56(1)$ \\
\hline $\mathrm{C}(3)$ & $6423(3)$ & $7240(2)$ & $7818(2)$ & $61(1)$ \\
\hline$C(25)$ & $13495(3)$ & $3563(2)$ & $9960(2)$ & $60(1)$ \\
\hline $\mathrm{C}(2)$ & $7546(3)$ & $7471(2)$ & $8183(2)$ & $65(1)$ \\
\hline $\mathrm{C}(24)$ & $13120(4)$ & $2955(2)$ & $9473(2)$ & $70(1)$ \\
\hline $\mathrm{C}(23)$ & $11784(4)$ & 2793(2) & $9361(2)$ & $72(1)$ \\
\hline C(33) & $6346(4)$ & $3581(2)$ & $8302(2)$ & $72(1)$ \\
\hline $\mathrm{B}(34)$ & $5152(4)$ & $5867(2)$ & $735(2)$ & $58(1)$ \\
\hline
\end{tabular}


Table 9S. Bond lengths $[\AA]$ and angles $\left[{ }^{\circ}\right]$ (compound 36).

\begin{tabular}{|c|c|}
\hline $\mathrm{Rh}(1)-\mathrm{C}(30)$ & $2.185(3)$ \\
\hline $\mathrm{Rh}(1)-\mathrm{C}(31)$ & $2.200(3)$ \\
\hline $\mathrm{Rh}(1)-\mathrm{C}(27)$ & $2.215(3)$ \\
\hline $\mathrm{Rh}(1)-\mathrm{C}(28)$ & $2.221(3)$ \\
\hline $\mathrm{Rh}(1)-\mathrm{P}(12)$ & $2.2731(6)$ \\
\hline $\mathrm{Rh}(1)-\mathrm{P}(15)$ & $2.3042(6)$ \\
\hline $\mathrm{P}(15)-\mathrm{C}(16)$ & $1.823(3)$ \\
\hline$P(15)-C(14)$ & $1.837(3)$ \\
\hline$P(15)-C(19)$ & $1.875(2)$ \\
\hline$C(16)-C(17)$ & $1.527(4)$ \\
\hline $\mathrm{C}(18)-\mathrm{C}(17)$ & $1.508(4)$ \\
\hline $\mathrm{C}(18)-\mathrm{C}(19)$ & $1.550(3)$ \\
\hline$C(19)-C(20)$ & $1.513(4)$ \\
\hline$C(20)-C(21)$ & $1.499(4)$ \\
\hline $\mathrm{P}(12)-\mathrm{C}(11)$ & $1.832(3)$ \\
\hline$P(12)-C(13)$ & $1.841(3)$ \\
\hline $\mathrm{P}(12)-\mathrm{C}(8)$ & $1.858(3)$ \\
\hline$C(27)-C(28)$ & $1.353(4)$ \\
\hline $\mathrm{C}(27)-\mathrm{C}(32)$ & $1.527(4)$ \\
\hline $\mathrm{F}(36)-\mathrm{B}(34)$ & $1.347(4)$ \\
\hline $\mathrm{F}(38)-\mathrm{B}(34)$ & $1.371(4)$ \\
\hline $\mathrm{C}(8)-\mathrm{C}(9)$ & $1.541(4)$ \\
\hline $\mathrm{C}(8)-\mathrm{C}(7)$ & $1.543(4)$ \\
\hline $\mathrm{F}(35)-\mathrm{B}(34)$ & $1.366(5)$ \\
\hline$C(31)-C(30)$ & $1.371(4)$ \\
\hline $\mathrm{C}(31)-\mathrm{C}(32)$ & $1.543(5)$ \\
\hline $\mathrm{F}(37)-\mathrm{B}(34)$ & $1.354(4)$ \\
\hline$C(13)-C(14)$ & $1.514(4)$ \\
\hline$C(10)-C(9)$ & $1.516(6)$ \\
\hline$C(10)-C(11)$ & $1.540(5)$ \\
\hline$C(6)-C(1)$ & $1.394(4)$ \\
\hline$C(6)-C(5)$ & $1.400(4)$ \\
\hline$C(6)-C(7)$ & $1.504(4)$ \\
\hline$C(21)-C(22)$ & $1.382(4)$ \\
\hline$C(21)-C(26)$ & $1.394(4)$ \\
\hline $\mathrm{C}(4)-\mathrm{C}(3)$ & $1.370(4)$ \\
\hline$C(4)-C(5)$ & $1.380(5)$ \\
\hline$C(22)-C(23)$ & $1.384(5)$ \\
\hline$C(26)-C(25)$ & $1.390(4)$ \\
\hline $\mathrm{C}(29)-\mathrm{C}(28)$ & $1.517(5)$ \\
\hline$C(29)-C(33)$ & $1.540(4)$ \\
\hline$C(29)-C(30)$ & $1.551(4)$ \\
\hline$C(1)-C(2)$ & $1.383(4)$ \\
\hline$C(32)-C(33)$ & $1.548(4)$ \\
\hline$C(3)-C(2)$ & $1.370(5)$ \\
\hline$C(25)-C(24)$ & $1.374(5)$ \\
\hline$C(24)-C(23)$ & $1.366(6)$ \\
\hline $\mathrm{C}(30)-\mathrm{Rh}(1)-\mathrm{C}(31)$ & $36.44(11)$ \\
\hline $\mathrm{C}(30)-\mathrm{Rh}(1)-\mathrm{C}(27)$ & $77.25(12)$ \\
\hline
\end{tabular}


Table 9S (continued).

\begin{tabular}{|c|c|}
\hline $\mathrm{C}(31)-\mathrm{Rh}(1)-\mathrm{C}(27)$ & $65.79(12)$ \\
\hline $\mathrm{C}(30)-\mathrm{Rh}(1)-\mathrm{C}(28)$ & $65.82(12)$ \\
\hline $\mathrm{C}(31)-\mathrm{Rh}(1)-\mathrm{C}(28)$ & $77.44(11)$ \\
\hline $\mathrm{C}(27)-\mathrm{Rh}(1)-\mathrm{C}(28)$ & $35.52(11)$ \\
\hline $\mathrm{C}(30)-\mathrm{Rh}(1)-\mathrm{P}(12)$ & $103.50(8)$ \\
\hline $\mathrm{C}(31)-\mathrm{Rh}(1)-\mathrm{P}(12)$ & $101.02(8)$ \\
\hline $\mathrm{C}(27)-\mathrm{Rh}(1)-\mathrm{P}(12)$ & $157.27(8)$ \\
\hline $\mathrm{C}(28)-\mathrm{Rh}(1)-\mathrm{P}(12)$ & $164.25(9)$ \\
\hline $\mathrm{C}(30)-\mathrm{Rh}(1)-\mathrm{P}(15)$ & $150.95(8)$ \\
\hline $\mathrm{C}(31)-\mathrm{Rh}(1)-\mathrm{P}(15)$ & $170.40(9)$ \\
\hline $\mathrm{C}(27)-\mathrm{Rh}(1)-\mathrm{P}(15)$ & $107.06(8)$ \\
\hline $\mathrm{C}(28)-\mathrm{Rh}(1)-\mathrm{P}(15)$ & $100.52(8)$ \\
\hline $\mathrm{P}(12)-\mathrm{Rh}(1)-\mathrm{P}(15)$ & $83.49(2)$ \\
\hline $\mathrm{C}(16)-\mathrm{P}(15)-\mathrm{C}(14)$ & $105.12(13)$ \\
\hline$C(16)-P(15)-C(19)$ & $94.94(12)$ \\
\hline $\mathrm{C}(14)-\mathrm{P}(15)-\mathrm{C}(19)$ & $102.55(12)$ \\
\hline $\mathrm{C}(16)-\mathrm{P}(15)-\mathrm{Rh}(1)$ & $121.35(9)$ \\
\hline $\mathrm{C}(14)-\mathrm{P}(15)-\mathrm{Rh}(1)$ & $109.26(8)$ \\
\hline $\mathrm{C}(19)-\mathrm{P}(15)-\mathrm{Rh}(1)$ & $121.04(8)$ \\
\hline $\mathrm{C}(17)-\mathrm{C}(16)-\mathrm{P}(15)$ & $105.68(18)$ \\
\hline $\mathrm{C}(17)-\mathrm{C}(18)-\mathrm{C}(19)$ & $108.3(2)$ \\
\hline $\mathrm{C}(20)-\mathrm{C}(19)-\mathrm{C}(18)$ & $113.8(2)$ \\
\hline $\mathrm{C}(20)-\mathrm{C}(19)-\mathrm{P}(15)$ & $117.9(2)$ \\
\hline $\mathrm{C}(18)-\mathrm{C}(19)-\mathrm{P}(15)$ & $103.64(17)$ \\
\hline$C(21)-C(20)-C(19)$ & $115.6(2)$ \\
\hline$C(18)-C(17)-C(16)$ & $106.6(2)$ \\
\hline $\mathrm{C}(11)-\mathrm{P}(12)-\mathrm{C}(13)$ & $105.93(16)$ \\
\hline $\mathrm{C}(11)-\mathrm{P}(12)-\mathrm{C}(8)$ & $95.60(14)$ \\
\hline $\mathrm{C}(13)-\mathrm{P}(12)-\mathrm{C}(8)$ & $101.29(13)$ \\
\hline $\mathrm{C}(11)-\mathrm{P}(12)-\mathrm{Rh}(1)$ & $122.81(12)$ \\
\hline $\mathrm{C}(13)-\mathrm{P}(12)-\mathrm{Rh}(1)$ & $109.60(8)$ \\
\hline $\mathrm{C}(8)-\mathrm{P}(12)-\mathrm{Rh}(1)$ & $118.81(9)$ \\
\hline $\mathrm{C}(28)-\mathrm{C}(27)-\mathrm{C}(32)$ & $107.0(3)$ \\
\hline $\mathrm{C}(28)-\mathrm{C}(27)-\mathrm{Rh}(1)$ & $72.49(16)$ \\
\hline $\mathrm{C}(32)-\mathrm{C}(27)-\mathrm{Rh}(1)$ & $95.58(18)$ \\
\hline $\mathrm{C}(9)-\mathrm{C}(8)-\mathrm{C}(7)$ & $111.4(2)$ \\
\hline $\mathrm{C}(9)-\mathrm{C}(8)-\mathrm{P}(12)$ & $102.72(19)$ \\
\hline $\mathrm{C}(7)-\mathrm{C}(8)-\mathrm{P}(12)$ & $114.87(19)$ \\
\hline$C(30)-C(31)-C(32)$ & $106.0(3)$ \\
\hline $\mathrm{C}(30)-\mathrm{C}(31)-\mathrm{Rh}(1)$ & $71.20(16)$ \\
\hline $\mathrm{C}(32)-\mathrm{C}(31)-\mathrm{Rh}(1)$ & $95.68(19)$ \\
\hline $\mathrm{C}(14)-\mathrm{C}(13)-\mathrm{P}(12)$ & $108.03(17)$ \\
\hline $\mathrm{C}(13)-\mathrm{C}(14)-\mathrm{P}(15)$ & $109.09(17)$ \\
\hline $\mathrm{C}(9)-\mathrm{C}(10)-\mathrm{C}(11)$ & $109.5(3)$ \\
\hline$C(1)-C(6)-C(5)$ & $117.2(3)$ \\
\hline$C(1)-C(6)-C(7)$ & $123.6(2)$ \\
\hline$C(5)-C(6)-C(7)$ & $118.9(2)$ \\
\hline$C(6)-C(7)-C(8)$ & $116.9(2)$ \\
\hline$C(22)-C(21)-C(26)$ & $118.2(3)$ \\
\hline$C(22)-C(21)-C(20)$ & $120.5(3)$ \\
\hline $\mathrm{C}(26)-\mathrm{C}(21)-\mathrm{C}(20)$ & $121.2(2)$ \\
\hline
\end{tabular}


Table 9S (continued).

$\begin{array}{lc}\mathrm{C}(10)-\mathrm{C}(9)-\mathrm{C}(8) & 109.1(2) \\ \mathrm{C}(3)-\mathrm{C}(4)-\mathrm{C}(5) & 120.7(3) \\ \mathrm{C}(21)-\mathrm{C}(22)-\mathrm{C}(23) & 121.0(3) \\ \mathrm{C}(25)-\mathrm{C}(26)-\mathrm{C}(21) & 120.2(3) \\ \mathrm{C}(4)-\mathrm{C}(5)-\mathrm{C}(6) & 120.9(3) \\ \mathrm{C}(28)-\mathrm{C}(29)-\mathrm{C}(33) & 100.2(3) \\ \mathrm{C}(28)-\mathrm{C}(29)-\mathrm{C}(30) & 102.6(2) \\ \mathrm{C}(33)-\mathrm{C}(29)-\mathrm{C}(30) & 99.9(3) \\ \mathrm{C}(2)-\mathrm{C}(1)-\mathrm{C}(6) & 121.2(3) \\ \mathrm{C}(31)-\mathrm{C}(30)-\mathrm{C}(29) & 106.6(3) \\ \mathrm{C}(31)-\mathrm{C}(30)-\mathrm{Rh}(1) & 72.36(16) \\ \mathrm{C}(29)-\mathrm{C}(30)-\mathrm{Rh}(1) & 95.83(18) \\ \mathrm{C}(27)-\mathrm{C}(32)-\mathrm{C}(31) & 102.7(2) \\ \mathrm{C}(27)-\mathrm{C}(32)-\mathrm{C}(33) & 99.7(3) \\ \mathrm{C}(31)-\mathrm{C}(32)-\mathrm{C}(33) & 100.3(3) \\ \mathrm{C}(10)-\mathrm{C}(11)-\mathrm{P}(12) & 106.9(2) \\ \mathrm{C}(27)-\mathrm{C}(28)-\mathrm{C}(29) & 106.9(2) \\ \mathrm{C}(27)-\mathrm{C}(28)-\mathrm{Rh}(1) & 71.99(16) \\ \mathrm{C}(29)-\mathrm{C}(28)-\mathrm{Rh}(1) & 95.43(17) \\ \mathrm{C}(4)-\mathrm{C}(3)-\mathrm{C}(2) & 119.7(3) \\ \mathrm{C}(24)-\mathrm{C}(25)-\mathrm{C}(26) & 120.5(3) \\ \mathrm{C}(3)-\mathrm{C}(2)-\mathrm{C}(1) & 120.3(3) \\ \mathrm{C}(23)-\mathrm{C}(24)-\mathrm{C}(25) & 119.6(3) \\ \mathrm{C}(24)-\mathrm{C}(23)-\mathrm{C}(22) & 120.5(3) \\ \mathrm{C}(29)-\mathrm{C}(33)-\mathrm{C}(32) & 93.0(2) \\ \mathrm{F}(36)-\mathrm{B}(34)-\mathrm{F}(37) & 113.7(3) \\ \mathrm{F}(36)-\mathrm{B}(34)-\mathrm{F}(35) & 106.5(3) \\ \mathrm{F}(37)-\mathrm{B}(34)-\mathrm{F}(35) & 107.0(3) \\ \mathrm{F}(36)-\mathrm{B}(34)-\mathrm{F}(38) & 111.3(3) \\ \mathrm{F}(37)-\mathrm{B}(34)-\mathrm{F}(38) & 111.8(3) \\ \mathrm{F}(35)-\mathrm{B}(34)-\mathrm{F}(38) & 106.1(3) \\ & \\ & \end{array}$


Table 10S. Anisotropic displacement parameters $\left(\AA^{2} \times 10^{3}\right)$. The anisotropic displacement factor exponent takes the form: $-2 \pi^{2}\left[h^{2} a^{* 2} U^{11}+\ldots+2 h k a^{*} b^{*} U^{12}\right]$ (compound 36).

\begin{tabular}{|c|c|c|c|c|c|c|}
\hline & $\mathrm{U}^{11}$ & $\mathrm{U}^{22}$ & $\mathrm{U}^{33}$ & $\mathrm{U}^{23}$ & $\mathrm{U}^{13}$ & $\mathrm{U}^{12}$ \\
\hline $\mathrm{Rh}(1)$ & $38(1)$ & $50(1)$ & $36(1)$ & $-11(1)$ & $6(1)$ & $-14(1)$ \\
\hline$P(15)$ & $34(1)$ & $33(1)$ & $30(1)$ & $-2(1)$ & $0(1)$ & $-4(1)$ \\
\hline$C(16)$ & $48(1)$ & $40(1)$ & $49(1)$ & $-3(1)$ & $-5(1)$ & $8(1)$ \\
\hline $\mathrm{C}(18)$ & $61(2)$ & $81(2)$ & $32(1)$ & $-1(1)$ & $4(1)$ & $26(2)$ \\
\hline C(19) & $37(1)$ & $45(1)$ & $33(1)$ & $1(1)$ & $1(1)$ & $7(1)$ \\
\hline$C(20)$ & $47(2)$ & $46(1)$ & $42(1)$ & $13(1)$ & $1(1)$ & $1(1)$ \\
\hline$C(17)$ & $59(2)$ & $60(2)$ & $47(1)$ & $-18(1)$ & $-3(1)$ & $17(1)$ \\
\hline $\mathrm{P}(12)$ & $36(1)$ & $39(1)$ & $34(1)$ & $3(1)$ & $5(1)$ & $-1(1)$ \\
\hline$C(27)$ & $41(1)$ & $62(2)$ & $72(2)$ & $-8(2)$ & $7(1)$ & $-8(1)$ \\
\hline $\mathrm{F}(36)$ & $135(2)$ & $59(1)$ & 131(2) & $3(1)$ & $-4(2)$ & $17(2)$ \\
\hline $\mathrm{F}(38)$ & $88(2)$ & $109(2)$ & 151(2) & $-57(2)$ & $-12(2)$ & $0(1)$ \\
\hline $\mathrm{C}(8)$ & $47(1)$ & $48(1)$ & $38(1)$ & $9(1)$ & $6(1)$ & $-5(1)$ \\
\hline $\mathrm{F}(35)$ & $136(3)$ & $156(3)$ & $109(2)$ & $45(2)$ & $34(2)$ & $31(2)$ \\
\hline $\mathrm{C}(31)$ & $65(2)$ & $54(2)$ & $36(1)$ & $-3(1)$ & $-5(1)$ & $-20(1)$ \\
\hline $\mathrm{F}(37)$ & $70(2)$ & $109(2)$ & $160(2)$ & $-38(2)$ & $34(2)$ & $-23(1)$ \\
\hline $\mathrm{C}(13)$ & $33(1)$ & $58(2)$ & 43(1) & $7(1)$ & $-3(1)$ & $-7(1)$ \\
\hline$C(14)$ & $44(1)$ & $49(2)$ & $43(1)$ & $-1(1)$ & $-3(1)$ & $-18(1)$ \\
\hline$C(10)$ & $65(2)$ & $98(3)$ & $60(2)$ & $1(2)$ & $27(1)$ & $22(2)$ \\
\hline $\mathrm{C}(6)$ & $50(2)$ & $42(1)$ & $35(1)$ & $6(1)$ & $-3(1)$ & $-2(1)$ \\
\hline $\mathrm{C}(7)$ & $55(2)$ & $50(1)$ & $36(1)$ & $5(1)$ & $3(1)$ & $0(1)$ \\
\hline $\mathrm{C}(21)$ & $50(1)$ & $33(1)$ & $41(1)$ & $10(1)$ & $-5(1)$ & $-1(1)$ \\
\hline $\mathrm{C}(9)$ & $45(2)$ & $89(2)$ & $51(2)$ & $15(2)$ & $15(1)$ & $-10(2)$ \\
\hline $\mathrm{C}(4)$ & $52(2)$ & $73(2)$ & $52(2)$ & $-4(2)$ & $-10(1)$ & $-6(2)$ \\
\hline$C(22)$ & $64(2)$ & $49(2)$ & $55(2)$ & $0(1)$ & $-9(2)$ & $-6(1)$ \\
\hline$C(26)$ & $51(2)$ & $38(1)$ & $57(2)$ & $3(1)$ & $-5(1)$ & $2(1)$ \\
\hline$C(5)$ & $63(2)$ & $55(2)$ & $36(1)$ & $-2(1)$ & $-3(1)$ & $-7(1)$ \\
\hline$C(29)$ & $62(2)$ & $56(2)$ & $61(2)$ & $17(2)$ & $-10(2)$ & $-22(1)$ \\
\hline $\mathrm{C}(1)$ & $55(2)$ & $59(2)$ & $47(2)$ & $-10(1)$ & $-12(1)$ & $6(1)$ \\
\hline$C(30)$ & $63(2)$ & $43(1)$ & $53(2)$ & $-7(1)$ & $4(1)$ & $-11(1)$ \\
\hline$C(32)$ & $60(2)$ & $67(2)$ & $57(2)$ & $14(2)$ & $-20(2)$ & $-16(2)$ \\
\hline $\mathrm{C}(11)$ & $72(2)$ & $54(2)$ & $66(2)$ & $-3(2)$ & $27(2)$ & $14(2)$ \\
\hline $\mathrm{C}(28)$ & $48(2)$ & $79(2)$ & $43(1)$ & $1(2)$ & $2(1)$ & $-25(2)$ \\
\hline $\mathrm{C}(3)$ & $55(2)$ & $74(2)$ & $54(2)$ & $-2(2)$ & $-4(1)$ & $10(2)$ \\
\hline$C(25)$ & $51(2)$ & $49(2)$ & $80(2)$ & $9(2)$ & $4(2)$ & $4(1)$ \\
\hline $\mathrm{C}(2)$ & $74(2)$ & $65(2)$ & $55(2)$ & $-17(2)$ & $-9(2)$ & $15(2)$ \\
\hline$C(24)$ & $94(3)$ & $53(2)$ & $64(2)$ & $8(2)$ & $24(2)$ & $23(2)$ \\
\hline $\mathrm{C}(23)$ & $98(3)$ & $59(2)$ & $60(2)$ & $-10(2)$ & $-6(2)$ & $8(2)$ \\
\hline$C(33)$ & $72(2)$ & $72(2)$ & $70(2)$ & $7(2)$ & $-19(2)$ & $-41(2)$ \\
\hline $\mathrm{B}(34)$ & $62(2)$ & $48(2)$ & $65(2)$ & $-3(2)$ & $10(2)$ & $1(2)$ \\
\hline
\end{tabular}


Table 11S. Hydrogen coordinates $\left(\begin{array}{ll}x & 10^{4}\end{array}\right)$ and isotropic displacement parameters $\left(\AA^{2} \mathrm{x} \quad 10{ }^{3}\right)$ (compound 36).

\begin{tabular}{|c|c|c|c|c|}
\hline & $\mathrm{X}$ & $\mathrm{y}$ & $\mathrm{z}$ & $\mathrm{U}(\mathrm{eq})$ \\
\hline $\mathrm{H}(16 \mathrm{~A})$ & 8948 & 7326 & 9786 & 55 \\
\hline $\mathrm{H}(16 \mathrm{~B})$ & 7795 & 6627 & 9923 & 55 \\
\hline $\mathrm{H}(18 \mathrm{~A})$ & 8288 & 5571 & 11006 & 69 \\
\hline $\mathrm{H}(18 \mathrm{~B})$ & 9495 & 5857 & 11476 & 69 \\
\hline $\mathrm{H}(19)$ & 11063 & 5557 & 10668 & 46 \\
\hline $\mathrm{H}(20 \mathrm{~A})$ & 10170 & 4212 & 11075 & 54 \\
\hline$H(20 B)$ & 9224 & 4167 & 10440 & 54 \\
\hline $\mathrm{H}(17 \mathrm{~A})$ & 9844 & 7142 & 10852 & 66 \\
\hline $\mathrm{H}(17 \mathrm{~B})$ & 8287 & 7110 & 11002 & 66 \\
\hline $\mathrm{H}(27)$ & 6374 & 5472 & 8921 & 70 \\
\hline $\mathrm{H}(8)$ & 11543 & 6783 & 7939 & 53 \\
\hline $\mathrm{H}(13 \mathrm{~A})$ & 12608 & 5307 & 9363 & 54 \\
\hline $\mathrm{H}(13 \mathrm{~B})$ & 13062 & 6095 & 8887 & 54 \\
\hline $\mathrm{H}(14 \mathrm{~A})$ & 11257 & 6958 & 9214 & 54 \\
\hline $\mathrm{H}(14 \mathrm{~B})$ & 11878 & 6589 & 9894 & 54 \\
\hline $\mathrm{H}(10 \mathrm{~A})$ & 13787 & 4763 & 7222 & 89 \\
\hline $\mathrm{H}(10 \mathrm{~B})$ & 12331 & 4759 & 6908 & 89 \\
\hline $\mathrm{H}(7 \mathrm{~A})$ & 10572 & 6634 & 6825 & 56 \\
\hline $\mathrm{H}(7 \mathrm{~B})$ & 10168 & 5656 & 7003 & 56 \\
\hline $\mathrm{H}(9 \mathrm{~A})$ & 12852 & 6231 & 6931 & 74 \\
\hline $\mathrm{H}(9 \mathrm{~B})$ & 13538 & 6158 & 7650 & 74 \\
\hline $\mathrm{H}(4)$ & 5783 & 6523 & 7022 & 71 \\
\hline $\mathrm{H}(22)$ & 9905 & 3149 & 9629 & 67 \\
\hline $\mathrm{H}(26)$ & 12791 & 4409 & 10677 & 58 \\
\hline $\mathrm{H}(5)$ & 7851 & 5969 & 6709 & 62 \\
\hline $\mathrm{H}(29)$ & 7579 & 2920 & 9061 & 72 \\
\hline $\mathrm{H}(1)$ & 9550 & 7305 & 8254 & 64 \\
\hline $\mathrm{H}(30)$ & 9424 & 3270 & 8209 & 64 \\
\hline $\mathrm{H}(11 \mathrm{~A})$ & 13055 & 4320 & 8241 & 77 \\
\hline $\mathrm{H}(11 \mathrm{~B})$ & 11794 & 3974 & 7833 & 77 \\
\hline $\mathrm{H}(28)$ & 7160 & 4337 & 9700 & 68 \\
\hline $\mathrm{H}(3)$ & 5584 & 7462 & 7941 & 73 \\
\hline $\mathrm{H}(25)$ & 14404 & 3674 & 10037 & 72 \\
\hline $\mathrm{H}(2)$ & 7466 & 7851 & 8555 & 77 \\
\hline $\mathrm{H}(24)$ & 13771 & 2656 & 9220 & 84 \\
\hline $\mathrm{H}(23)$ & 11528 & 2370 & 9040 & 86 \\
\hline$H(33 A)$ & 5472 & 3574 & 8523 & 86 \\
\hline $\mathrm{H}(33 \mathrm{~B})$ & 6372 & 3150 & 7934 & 86 \\
\hline $\mathrm{H}(32)$ & $6210(40)$ & $4780(20)$ & 7761(19) & $76(10)$ \\
\hline $\mathrm{H}(31)$ & $8680(30)$ & $4417(18)$ & $7447(15)$ & $48(8)$ \\
\hline
\end{tabular}


Table 12S. Torsion angles $\left[{ }^{\circ}\right]$ (compound 36).

\begin{tabular}{|c|c|}
\hline $\mathrm{C}(30)-\mathrm{Rh}(1)-\mathrm{P}(15)-\mathrm{C}(16)$ & $-122.3(2)$ \\
\hline $\mathrm{C}(31)-\mathrm{Rh}(1)-\mathrm{P}(15)-\mathrm{C}(16)$ & $12.9(5)$ \\
\hline $\mathrm{C}(27)-\mathrm{Rh}(1)-\mathrm{P}(15)-\mathrm{C}(16)$ & $-27.91(14)$ \\
\hline $\mathrm{C}(28)-\mathrm{Rh}(1)-\mathrm{P}(15)-\mathrm{C}(16)$ & $-63.91(14)$ \\
\hline $\mathrm{P}(12)-\mathrm{Rh}(1)-\mathrm{P}(15)-\mathrm{C}(16)$ & 131.49(11) \\
\hline $\mathrm{C}(30)-\mathrm{Rh}(1)-\mathrm{P}(15)-\mathrm{C}(14)$ & $115.2(2)$ \\
\hline $\mathrm{C}(31)-\mathrm{Rh}(1)-\mathrm{P}(15)-\mathrm{C}(14)$ & $-109.5(5)$ \\
\hline $\mathrm{C}(27)-\mathrm{Rh}(1)-\mathrm{P}(15)-\mathrm{C}(14)$ & $-150.34(13)$ \\
\hline $\mathrm{C}(28)-\mathrm{Rh}(1)-\mathrm{P}(15)-\mathrm{C}(14)$ & 173.66(13) \\
\hline $\mathrm{P}(12)-\mathrm{Rh}(1)-\mathrm{P}(15)-\mathrm{C}(14)$ & $9.06(10)$ \\
\hline $\mathrm{C}(30)-\mathrm{Rh}(1)-\mathrm{P}(15)-\mathrm{C}(19)$ & $-3.4(2)$ \\
\hline $\mathrm{C}(31)-\mathrm{Rh}(1)-\mathrm{P}(15)-\mathrm{C}(19)$ & $131.8(5)$ \\
\hline $\mathrm{C}(27)-\mathrm{Rh}(1)-\mathrm{P}(15)-\mathrm{C}(19)$ & $91.05(13)$ \\
\hline $\mathrm{C}(28)-\mathrm{Rh}(1)-\mathrm{P}(15)-\mathrm{C}(19)$ & $55.05(13)$ \\
\hline $\mathrm{P}(12)-\mathrm{Rh}(1)-\mathrm{P}(15)-\mathrm{C}(19)$ & $-109.55(10)$ \\
\hline $\mathrm{C}(14)-\mathrm{P}(15)-\mathrm{C}(16)-\mathrm{C}(17)$ & $-87.5(2)$ \\
\hline $\mathrm{C}(19)-\mathrm{P}(15)-\mathrm{C}(16)-\mathrm{C}(17)$ & $16.9(2)$ \\
\hline $\mathrm{Rh}(1)-\mathrm{P}(15)-\mathrm{C}(16)-\mathrm{C}(17)$ & $148.13(17)$ \\
\hline$C(17)-C(18)-C(19)-C(20)$ & $-164.1(3)$ \\
\hline $\mathrm{C}(17)-\mathrm{C}(18)-\mathrm{C}(19)-\mathrm{P}(15)$ & $-34.8(3)$ \\
\hline $\mathrm{C}(16)-\mathrm{P}(15)-\mathrm{C}(19)-\mathrm{C}(20)$ & $136.4(2)$ \\
\hline $\mathrm{C}(14)-\mathrm{P}(15)-\mathrm{C}(19)-\mathrm{C}(20)$ & $-116.9(2)$ \\
\hline $\mathrm{Rh}(1)-\mathrm{P}(15)-\mathrm{C}(19)-\mathrm{C}(20)$ & $5.0(3)$ \\
\hline $\mathrm{C}(16)-\mathrm{P}(15)-\mathrm{C}(19)-\mathrm{C}(18)$ & $9.7(2)$ \\
\hline $\mathrm{C}(14)-\mathrm{P}(15)-\mathrm{C}(19)-\mathrm{C}(18)$ & $116.4(2)$ \\
\hline $\mathrm{Rh}(1)-\mathrm{P}(15)-\mathrm{C}(19)-\mathrm{C}(18)$ & $-121.72(18)$ \\
\hline$C(18)-C(19)-C(20)-C(21)$ & $-172.4(2)$ \\
\hline$P(15)-C(19)-C(20)-C(21)$ & $65.9(3)$ \\
\hline$C(19)-C(18)-C(17)-C(16)$ & $49.7(3)$ \\
\hline$P(15)-C(16)-C(17)-C(18)$ & $-40.0(3)$ \\
\hline $\mathrm{C}(30)-\mathrm{Rh}(1)-\mathrm{P}(12)-\mathrm{C}(11)$ & $-11.36(17)$ \\
\hline $\mathrm{C}(31)-\mathrm{Rh}(1)-\mathrm{P}(12)-\mathrm{C}(11)$ & $-48.60(17)$ \\
\hline $\mathrm{C}(27)-\mathrm{Rh}(1)-\mathrm{P}(12)-\mathrm{C}(11)$ & $-100.5(3)$ \\
\hline $\mathrm{C}(28)-\mathrm{Rh}(1)-\mathrm{P}(12)-\mathrm{C}(11)$ & $34.2(3)$ \\
\hline $\mathrm{P}(15)-\mathrm{Rh}(1)-\mathrm{P}(12)-\mathrm{C}(11)$ & $139.98(15)$ \\
\hline $\mathrm{C}(30)-\mathrm{Rh}(1)-\mathrm{P}(12)-\mathrm{C}(13)$ & $-136.54(13)$ \\
\hline $\mathrm{C}(31)-\mathrm{Rh}(1)-\mathrm{P}(12)-\mathrm{C}(13)$ & $-173.78(13)$ \\
\hline $\mathrm{C}(27)-\mathrm{Rh}(1)-\mathrm{P}(12)-\mathrm{C}(13)$ & $134.3(2)$ \\
\hline $\mathrm{C}(28)-\mathrm{Rh}(1)-\mathrm{P}(12)-\mathrm{C}(13)$ & $-91.0(3)$ \\
\hline $\mathrm{P}(15)-\mathrm{Rh}(1)-\mathrm{P}(12)-\mathrm{C}(13)$ & $14.80(10)$ \\
\hline $\mathrm{C}(30)-\mathrm{Rh}(1)-\mathrm{P}(12)-\mathrm{C}(8)$ & $107.80(13)$ \\
\hline $\mathrm{C}(31)-\mathrm{Rh}(1)-\mathrm{P}(12)-\mathrm{C}(8)$ & $70.56(13)$ \\
\hline $\mathrm{C}(27)-\mathrm{Rh}(1)-\mathrm{P}(12)-\mathrm{C}(8)$ & $18.6(2)$ \\
\hline $\mathrm{C}(28)-\mathrm{Rh}(1)-\mathrm{P}(12)-\mathrm{C}(8)$ & $153.3(3)$ \\
\hline $\mathrm{P}(15)-\mathrm{Rh}(1)-\mathrm{P}(12)-\mathrm{C}(8)$ & $-100.86(10)$ \\
\hline $\mathrm{C}(30)-\mathrm{Rh}(1)-\mathrm{C}(27)-\mathrm{C}(28)$ & $66.06(19)$ \\
\hline $\mathrm{C}(31)-\mathrm{Rh}(1)-\mathrm{C}(27)-\mathrm{C}(28)$ & $102.7(2)$ \\
\hline $\mathrm{P}(12)-\mathrm{Rh}(1)-\mathrm{C}(27)-\mathrm{C}(28)$ & $160.61(18)$ \\
\hline $\mathrm{P}(15)-\mathrm{Rh}(1)-\mathrm{C}(27)-\mathrm{C}(28)$ & $-84.18(18)$ \\
\hline $\mathrm{C}(30)-\mathrm{Rh}(1)-\mathrm{C}(27)-\mathrm{C}(32)$ & $-40.0(2)$ \\
\hline
\end{tabular}




\begin{tabular}{|c|c|}
\hline $\mathrm{C}(31)-\mathrm{Rh}(1)-\mathrm{C}(27)-\mathrm{C}(32)$ & $-3.39(18)$ \\
\hline $\mathrm{C}(28)-\mathrm{Rh}(1)-\mathrm{C}(27)-\mathrm{C}(32)$ & $-106.1(3)$ \\
\hline $\mathrm{P}(12)-\mathrm{Rh}(1)-\mathrm{C}(27)-\mathrm{C}(32)$ & $54.5(3)$ \\
\hline $\mathrm{P}(15)-\mathrm{Rh}(1)-\mathrm{C}(27)-\mathrm{C}(32)$ & $169.75(17)$ \\
\hline $\mathrm{C}(11)-\mathrm{P}(12)-\mathrm{C}(8)-\mathrm{C}(9)$ & $-24.1(2)$ \\
\hline $\mathrm{C}(13)-\mathrm{P}(12)-\mathrm{C}(8)-\mathrm{C}(9)$ & $83.4(2)$ \\
\hline $\mathrm{Rh}(1)-\mathrm{P}(12)-\mathrm{C}(8)-\mathrm{C}(9)$ & $-156.56(17)$ \\
\hline $\mathrm{C}(11)-\mathrm{P}(12)-\mathrm{C}(8)-\mathrm{C}(7)$ & $97.0(2)$ \\
\hline $\mathrm{C}(13)-\mathrm{P}(12)-\mathrm{C}(8)-\mathrm{C}(7)$ & $-155.5(2)$ \\
\hline $\mathrm{Rh}(1)-\mathrm{P}(12)-\mathrm{C}(8)-\mathrm{C}(7)$ & $-35.5(2)$ \\
\hline $\mathrm{C}(27)-\mathrm{Rh}(1)-\mathrm{C}(31)-\mathrm{C}(30)$ & $-101.6(2)$ \\
\hline $\mathrm{C}(28)-\mathrm{Rh}(1)-\mathrm{C}(31)-\mathrm{C}(30)$ & $-66.1(2)$ \\
\hline $\mathrm{P}(12)-\mathrm{Rh}(1)-\mathrm{C}(31)-\mathrm{C}(30)$ & $97.86(18)$ \\
\hline $\mathrm{P}(15)-\mathrm{Rh}(1)-\mathrm{C}(31)-\mathrm{C}(30)$ & $-144.8(4)$ \\
\hline $\mathrm{C}(30)-\mathrm{Rh}(1)-\mathrm{C}(31)-\mathrm{C}(32)$ & $105.0(3)$ \\
\hline $\mathrm{C}(27)-\mathrm{Rh}(1)-\mathrm{C}(31)-\mathrm{C}(32)$ & $3.36(18)$ \\
\hline $\mathrm{C}(28)-\mathrm{Rh}(1)-\mathrm{C}(31)-\mathrm{C}(32)$ & $38.85(19)$ \\
\hline $\mathrm{P}(12)-\mathrm{Rh}(1)-\mathrm{C}(31)-\mathrm{C}(32)$ & $-157.16(16)$ \\
\hline $\mathrm{P}(15)-\mathrm{Rh}(1)-\mathrm{C}(31)-\mathrm{C}(32)$ & $-39.9(6)$ \\
\hline $\mathrm{C}(11)-\mathrm{P}(12)-\mathrm{C}(13)-\mathrm{C}(14)$ & $-175.85(18)$ \\
\hline $\mathrm{C}(8)-\mathrm{P}(12)-\mathrm{C}(13)-\mathrm{C}(14)$ & 84.91(19) \\
\hline $\mathrm{Rh}(1)-\mathrm{P}(12)-\mathrm{C}(13)-\mathrm{C}(14)$ & $-41.44(19)$ \\
\hline $\mathrm{P}(12)-\mathrm{C}(13)-\mathrm{C}(14)-\mathrm{P}(15)$ & $48.9(2)$ \\
\hline $\mathrm{C}(16)-\mathrm{P}(15)-\mathrm{C}(14)-\mathrm{C}(13)$ & $-168.64(19)$ \\
\hline C(19)-P(15)-C(14)-C(13) & $92.65(19)$ \\
\hline $\mathrm{Rh}(1)-\mathrm{P}(15)-\mathrm{C}(14)-\mathrm{C}(13)$ & $-36.9(2)$ \\
\hline$C(1)-C(6)-C(7)-C(8)$ & $26.8(4)$ \\
\hline$C(5)-C(6)-C(7)-C(8)$ & $-158.4(3)$ \\
\hline $\mathrm{C}(9)-\mathrm{C}(8)-\mathrm{C}(7)-\mathrm{C}(6)$ & $-174.4(2)$ \\
\hline$P(12)-C(8)-C(7)-C(6)$ & $69.3(3)$ \\
\hline$C(19)-C(20)-C(21)-C(22)$ & $-120.7(3)$ \\
\hline$C(19)-C(20)-C(21)-C(26)$ & $58.9(3)$ \\
\hline $\mathrm{C}(11)-\mathrm{C}(10)-\mathrm{C}(9)-\mathrm{C}(8)$ & $-41.4(4)$ \\
\hline$C(7)-C(8)-C(9)-C(10)$ & $-83.0(3)$ \\
\hline $\mathrm{P}(12)-\mathrm{C}(8)-\mathrm{C}(9)-\mathrm{C}(10)$ & $40.4(3)$ \\
\hline$C(26)-C(21)-C(22)-C(23)$ & $0.0(4)$ \\
\hline$C(20)-C(21)-C(22)-C(23)$ & $179.6(3)$ \\
\hline$C(22)-C(21)-C(26)-C(25)$ & $1.9(4)$ \\
\hline$C(20)-C(21)-C(26)-C(25)$ & $-177.8(2)$ \\
\hline$C(3)-C(4)-C(5)-C(6)$ & $0.2(5)$ \\
\hline$C(1)-C(6)-C(5)-C(4)$ & $-0.3(4)$ \\
\hline$C(7)-C(6)-C(5)-C(4)$ & $-175.4(3)$ \\
\hline $\mathrm{C}(5)-\mathrm{C}(6)-\mathrm{C}(1)-\mathrm{C}(2)$ & $0.2(4)$ \\
\hline $\mathrm{C}(7)-\mathrm{C}(6)-\mathrm{C}(1)-\mathrm{C}(2)$ & $175.1(3)$ \\
\hline$C(32)-C(31)-C(30)-C(29)$ & $0.3(3)$ \\
\hline $\mathrm{Rh}(1)-\mathrm{C}(31)-\mathrm{C}(30)-\mathrm{C}(29)$ & $90.9(2)$ \\
\hline $\mathrm{C}(32)-\mathrm{C}(31)-\mathrm{C}(30)-\mathrm{Rh}(1)$ & $-90.6(2)$ \\
\hline$C(28)-C(29)-C(30)-C(31)$ & $-68.2(3)$ \\
\hline$C(33)-C(29)-C(30)-C(31)$ & $34.7(3)$ \\
\hline$C(28)-C(29)-C(30)-R h(1)$ & $5.1(2)$ \\
\hline $\mathrm{C}(33)-\mathrm{C}(29)-\mathrm{C}(30)-\mathrm{Rh}(1)$ & $108.0(2)$ \\
\hline $\mathrm{C}(27)-\mathrm{Rh}(1)-\mathrm{C}(30)-\mathrm{C}(31)$ & $66.3(2)$ \\
\hline
\end{tabular}




\begin{tabular}{|c|c|}
\hline $\mathrm{C}(28)-\mathrm{Rh}(1)-\mathrm{C}(30)-\mathrm{C}(31)$ & $101.9(2)$ \\
\hline $\mathrm{P}(12)-\mathrm{Rh}(1)-\mathrm{C}(30)-\mathrm{C}(31)$ & $-90.33(18)$ \\
\hline $\mathrm{P}(15)-\mathrm{Rh}(1)-\mathrm{C}(30)-\mathrm{C}(31)$ & $168.59(15)$ \\
\hline $\mathrm{C}(31)-\mathrm{Rh}(1)-\mathrm{C}(30)-\mathrm{C}(29)$ & $-105.6(3)$ \\
\hline $\mathrm{C}(27)-\mathrm{Rh}(1)-\mathrm{C}(30)-\mathrm{C}(29)$ & $-39.30(18)$ \\
\hline $\mathrm{C}(28)-\mathrm{Rh}(1)-\mathrm{C}(30)-\mathrm{C}(29)$ & $-3.71(17)$ \\
\hline $\mathrm{P}(12)-\mathrm{Rh}(1)-\mathrm{C}(30)-\mathrm{C}(29)$ & $164.03(16)$ \\
\hline $\mathrm{P}(15)-\mathrm{Rh}(1)-\mathrm{C}(30)-\mathrm{C}(29)$ & $62.9(3)$ \\
\hline $\mathrm{C}(28)-\mathrm{C}(27)-\mathrm{C}(32)-\mathrm{C}(31)$ & $-68.8(3)$ \\
\hline $\mathrm{Rh}(1)-\mathrm{C}(27)-\mathrm{C}(32)-\mathrm{C}(31)$ & $4.5(2)$ \\
\hline $\mathrm{C}(28)-\mathrm{C}(27)-\mathrm{C}(32)-\mathrm{C}(33)$ & $34.1(3)$ \\
\hline $\mathrm{Rh}(1)-\mathrm{C}(27)-\mathrm{C}(32)-\mathrm{C}(33)$ & $107.5(2)$ \\
\hline$C(30)-C(31)-C(32)-C(27)$ & $67.5(3)$ \\
\hline $\mathrm{Rh}(1)-\mathrm{C}(31)-\mathrm{C}(32)-\mathrm{C}(27)$ & $-4.6(2)$ \\
\hline $\mathrm{C}(30)-\mathrm{C}(31)-\mathrm{C}(32)-\mathrm{C}(33)$ & $-35.1(3)$ \\
\hline $\mathrm{Rh}(1)-\mathrm{C}(31)-\mathrm{C}(32)-\mathrm{C}(33)$ & $-107.1(2)$ \\
\hline $\mathrm{C}(9)-\mathrm{C}(10)-\mathrm{C}(11)-\mathrm{P}(12)$ & $21.2(4)$ \\
\hline $\mathrm{C}(13)-\mathrm{P}(12)-\mathrm{C}(11)-\mathrm{C}(10)$ & $-101.1(3)$ \\
\hline $\mathrm{C}(8)-\mathrm{P}(12)-\mathrm{C}(11)-\mathrm{C}(10)$ & $2.3(3)$ \\
\hline $\mathrm{Rh}(1)-\mathrm{P}(12)-\mathrm{C}(11)-\mathrm{C}(10)$ & $132.1(2)$ \\
\hline $\mathrm{C}(32)-\mathrm{C}(27)-\mathrm{C}(28)-\mathrm{C}(29)$ & $0.3(3)$ \\
\hline $\mathrm{Rh}(1)-\mathrm{C}(27)-\mathrm{C}(28)-\mathrm{C}(29)$ & $-90.3(2)$ \\
\hline $\mathrm{C}(32)-\mathrm{C}(27)-\mathrm{C}(28)-\mathrm{Rh}(1)$ & $90.6(2)$ \\
\hline $\mathrm{C}(33)-\mathrm{C}(29)-\mathrm{C}(28)-\mathrm{C}(27)$ & $-34.9(3)$ \\
\hline $\mathrm{C}(30)-\mathrm{C}(29)-\mathrm{C}(28)-\mathrm{C}(27)$ & $67.8(3)$ \\
\hline $\mathrm{C}(33)-\mathrm{C}(29)-\mathrm{C}(28)-\mathrm{Rh}(1)$ & $-107.7(2)$ \\
\hline $\mathrm{C}(30)-\mathrm{C}(29)-\mathrm{C}(28)-\mathrm{Rh}(1)$ & $-5.0(2)$ \\
\hline $\mathrm{C}(30)-\mathrm{Rh}(1)-\mathrm{C}(28)-\mathrm{C}(27)$ & $-102.3(2)$ \\
\hline $\mathrm{C}(31)-\mathrm{Rh}(1)-\mathrm{C}(28)-\mathrm{C}(27)$ & $-65.73(19)$ \\
\hline $\mathrm{P}(12)-\mathrm{Rh}(1)-\mathrm{C}(28)-\mathrm{C}(27)$ & $-151.8(2)$ \\
\hline $\mathrm{P}(15)-\mathrm{Rh}(1)-\mathrm{C}(28)-\mathrm{C}(27)$ & 104.69(17) \\
\hline $\mathrm{C}(30)-\mathrm{Rh}(1)-\mathrm{C}(28)-\mathrm{C}(29)$ & $3.79(18)$ \\
\hline $\mathrm{C}(31)-\mathrm{Rh}(1)-\mathrm{C}(28)-\mathrm{C}(29)$ & $40.33(18)$ \\
\hline $\mathrm{C}(27)-\mathrm{Rh}(1)-\mathrm{C}(28)-\mathrm{C}(29)$ & $106.1(2)$ \\
\hline $\mathrm{P}(12)-\mathrm{Rh}(1)-\mathrm{C}(28)-\mathrm{C}(29)$ & $-45.7(4)$ \\
\hline $\mathrm{P}(15)-\mathrm{Rh}(1)-\mathrm{C}(28)-\mathrm{C}(29)$ & $-149.25(16)$ \\
\hline$C(5)-C(4)-C(3)-C(2)$ & $0.0(5)$ \\
\hline $\mathrm{C}(21)-\mathrm{C}(26)-\mathrm{C}(25)-\mathrm{C}(24)$ & $-1.9(4)$ \\
\hline $\mathrm{C}(4)-\mathrm{C}(3)-\mathrm{C}(2)-\mathrm{C}(1)$ & $0.0(5)$ \\
\hline$C(6)-C(1)-C(2)-C(3)$ & $-0.1(5)$ \\
\hline$C(26)-C(25)-C(24)-C(23)$ & $0.0(5)$ \\
\hline $\mathrm{C}(25)-\mathrm{C}(24)-\mathrm{C}(23)-\mathrm{C}(22)$ & $1.8(5)$ \\
\hline $\mathrm{C}(21)-\mathrm{C}(22)-\mathrm{C}(23)-\mathrm{C}(24)$ & $-1.8(5)$ \\
\hline $\mathrm{C}(28)-\mathrm{C}(29)-\mathrm{C}(33)-\mathrm{C}(32)$ & $52.2(3)$ \\
\hline$C(30)-C(29)-C(33)-C(32)$ & $-52.7(3)$ \\
\hline $\mathrm{C}(27)-\mathrm{C}(32)-\mathrm{C}(33)-\mathrm{C}(29)$ & $-51.7(3)$ \\
\hline $\mathrm{C}(31)-\mathrm{C}(32)-\mathrm{C}(33)-\mathrm{C}(29)$ & $53.2(3)$ \\
\hline
\end{tabular}




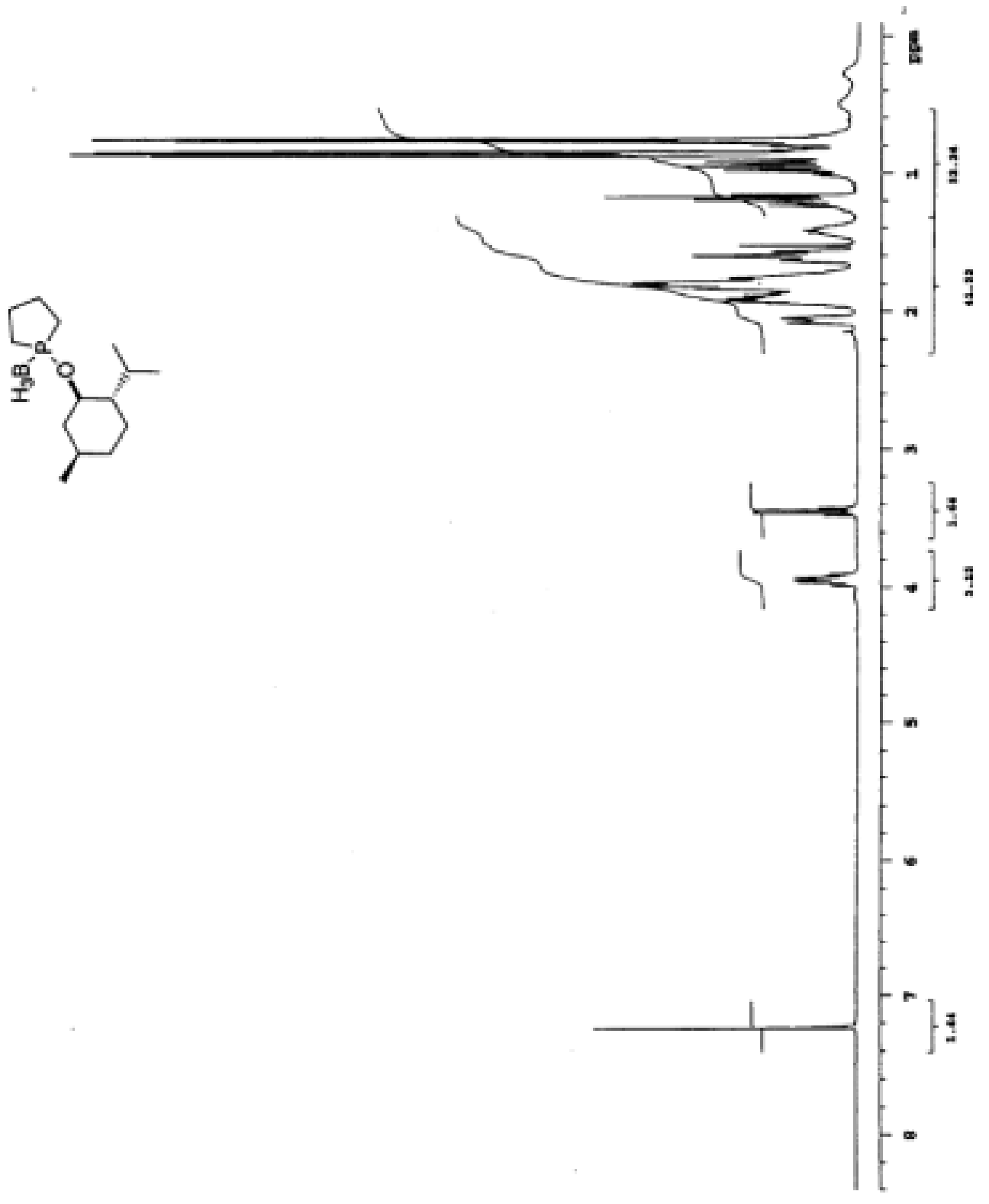

Figure 6S. ${ }^{1} \mathrm{H}$ NMR of compound $21\left(400 \mathrm{MHz}, \mathrm{CDCl}_{3}\right)$. 

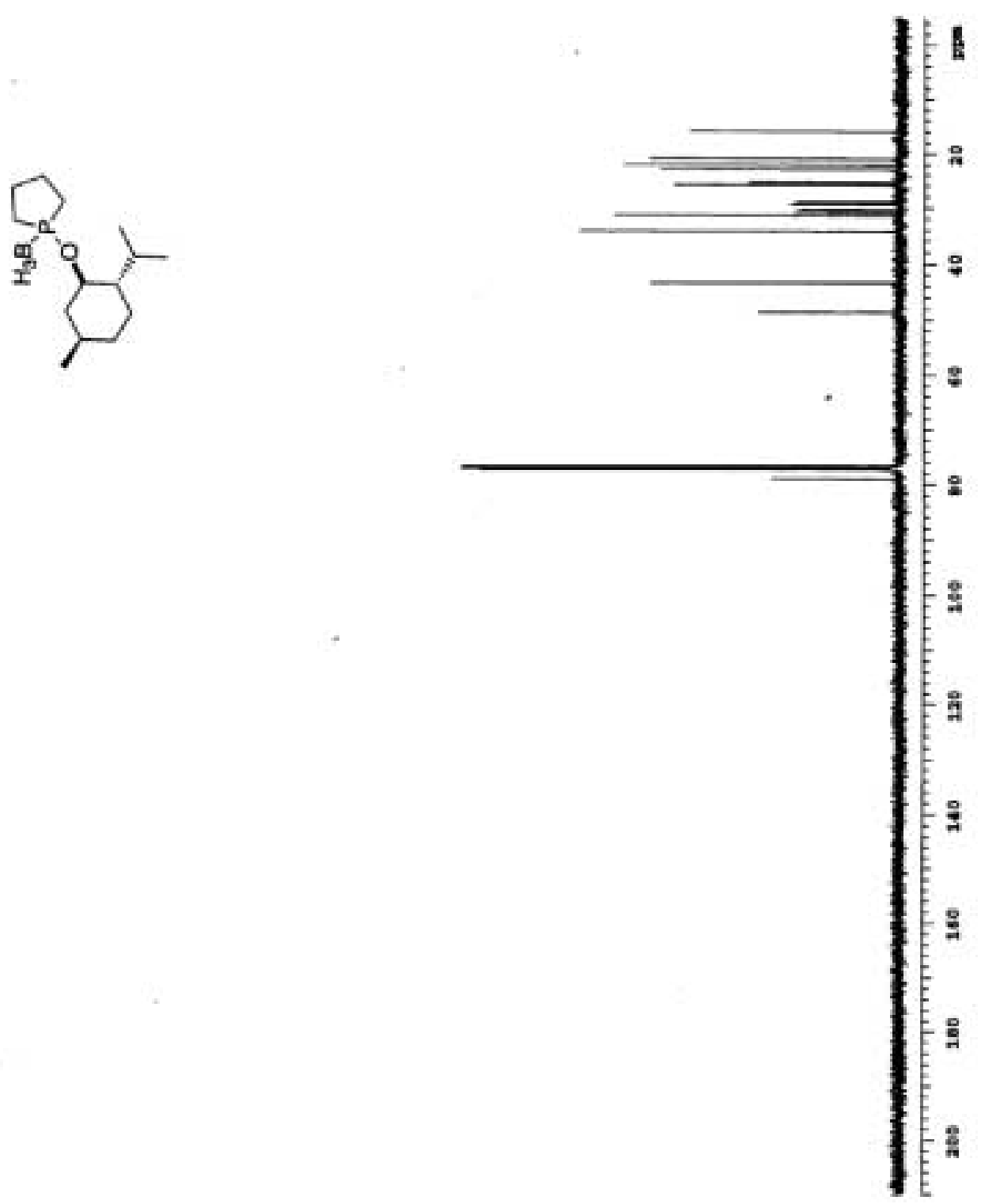

Figure 7S. ${ }^{13} \mathrm{C}$ NMR of compound $21\left(100 \mathrm{MHz}, \mathrm{CDCl}_{3}\right)$. 


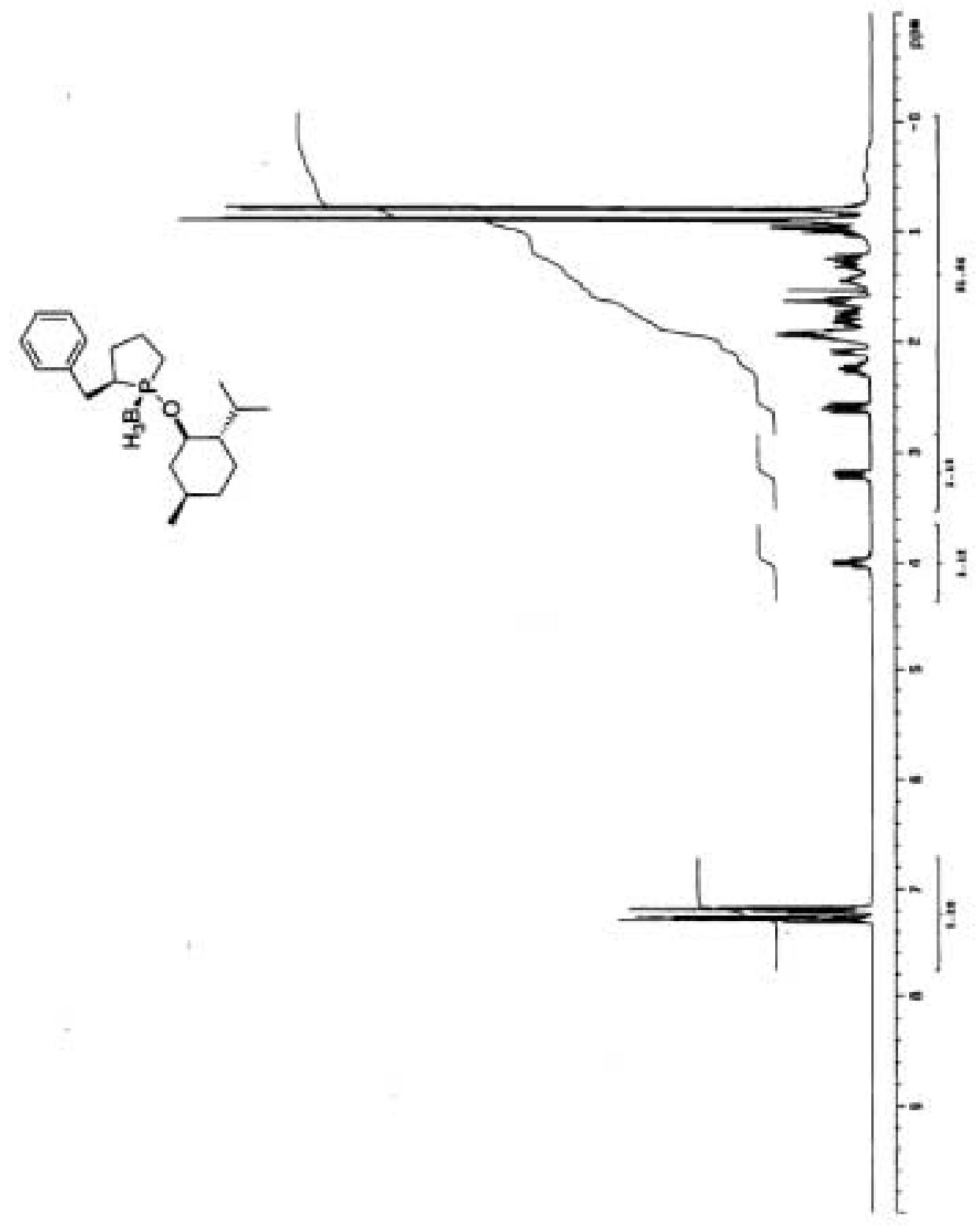

Figure 8S. ${ }^{1} \mathrm{H} \mathrm{NMR}$ of compound $23\left(400 \mathrm{MHz}, \mathrm{CDCl}_{3}\right)$. 

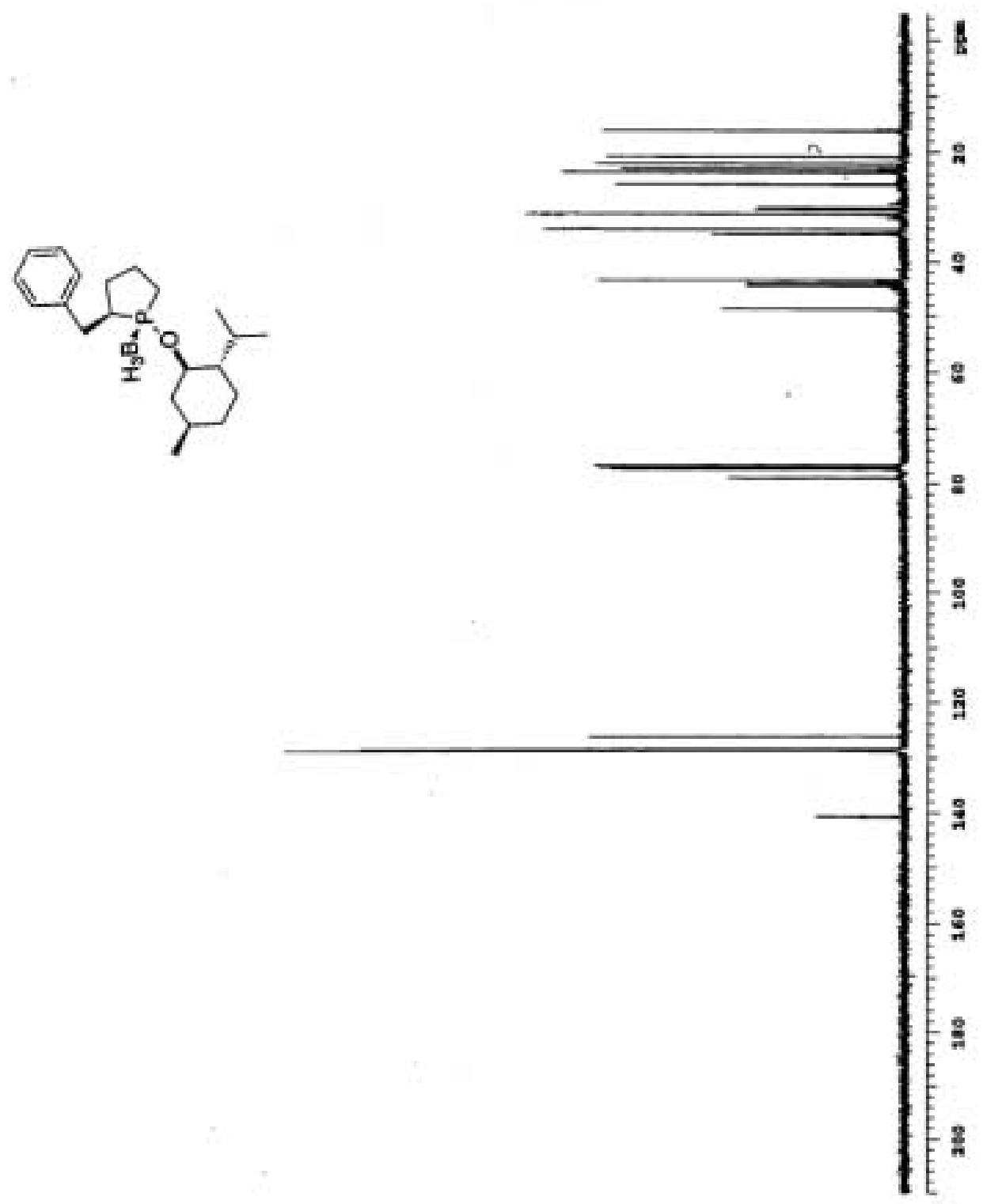

Figure 9S. ${ }^{13} \mathrm{C}$ NMR of compound $23\left(100 \mathrm{MHz}, \mathrm{CDCl}_{3}\right)$. 


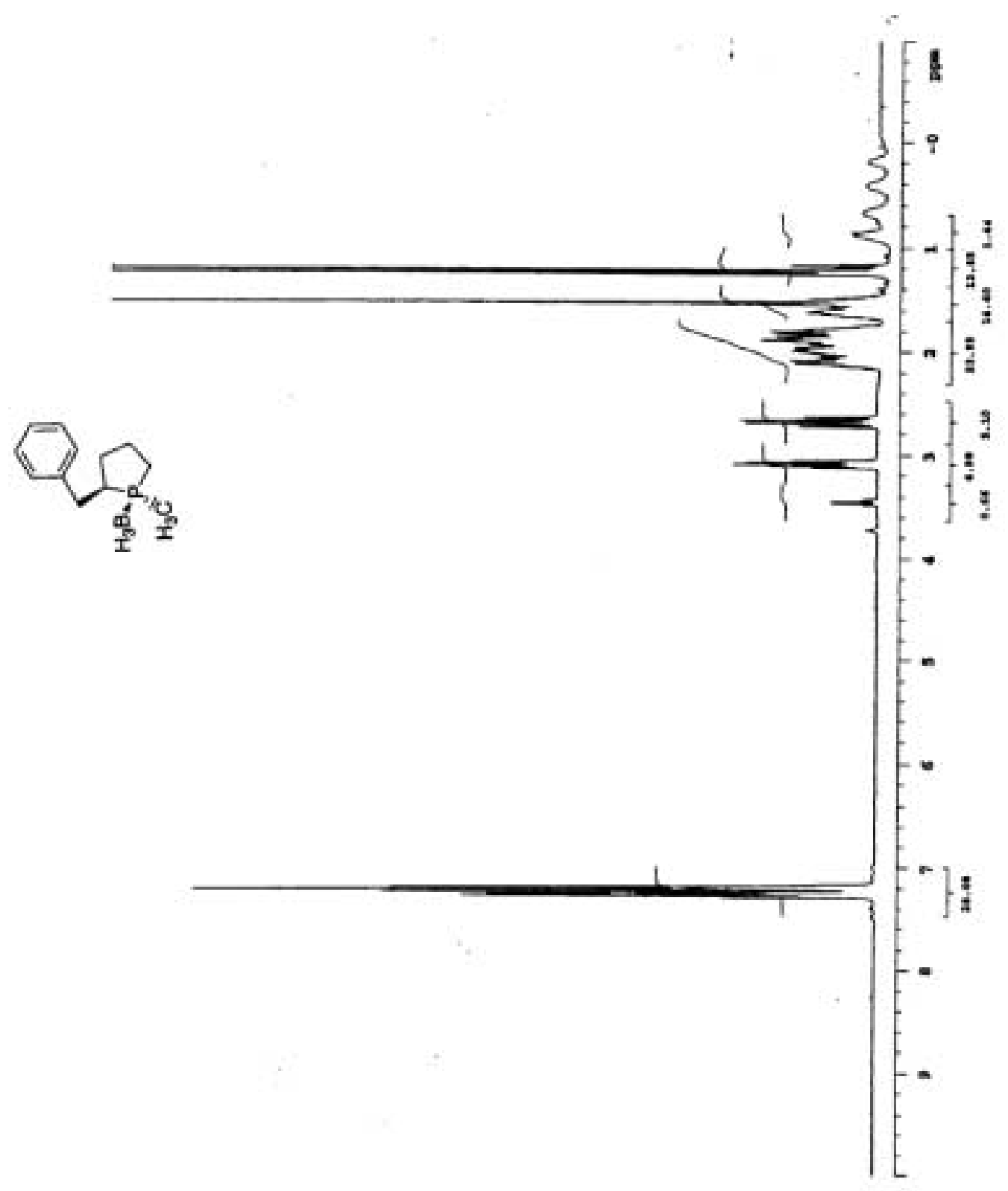

Figure 10S. ${ }^{1} \mathrm{H}$ NMR of compound $20\left(400 \mathrm{MHz}, \mathrm{CDCl}_{3}\right)$. 


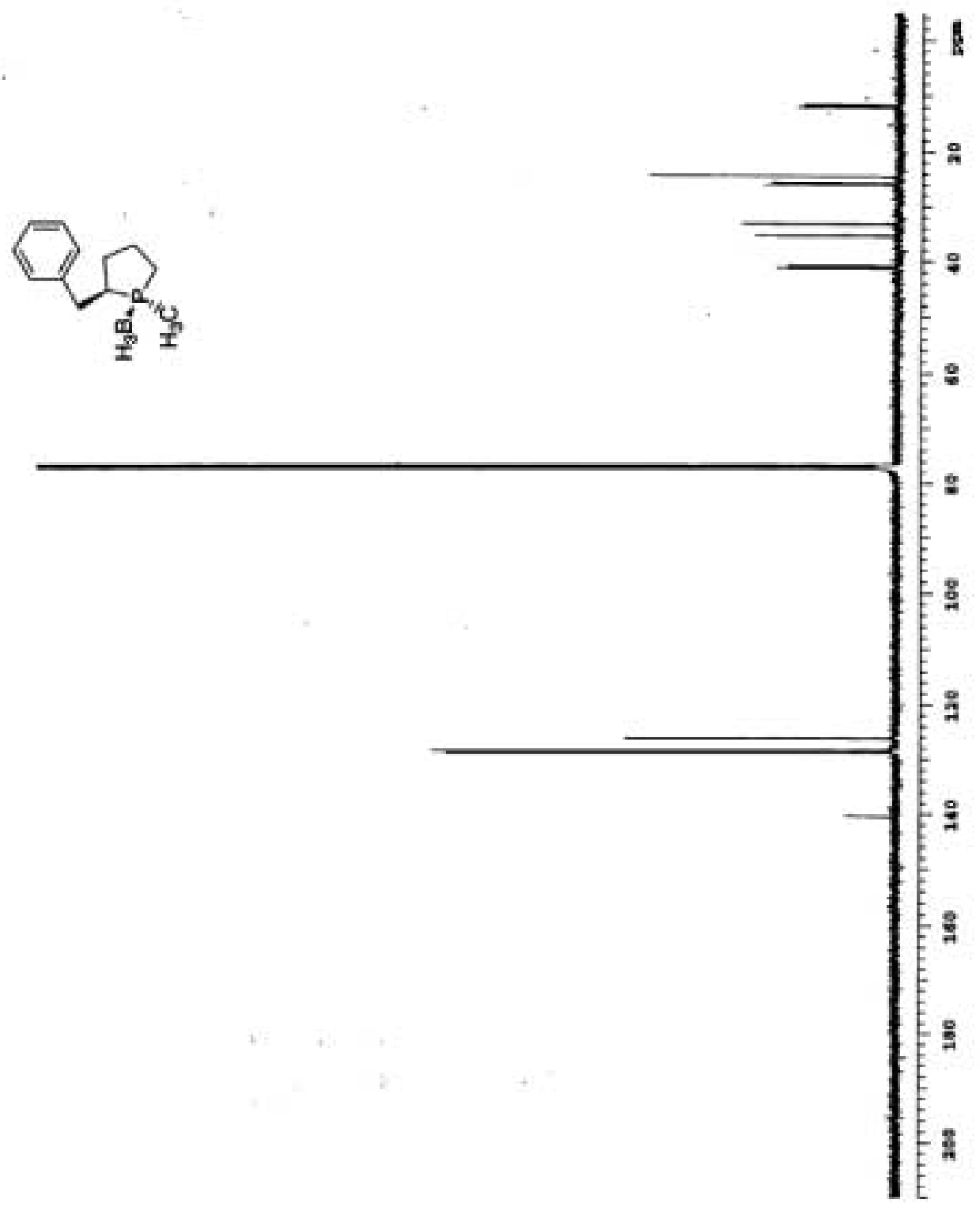

Figure 11S. ${ }^{13} \mathrm{C}$ NMR of compound $20\left(100 \mathrm{MHz}, \mathrm{CDCl}_{3}\right)$. 

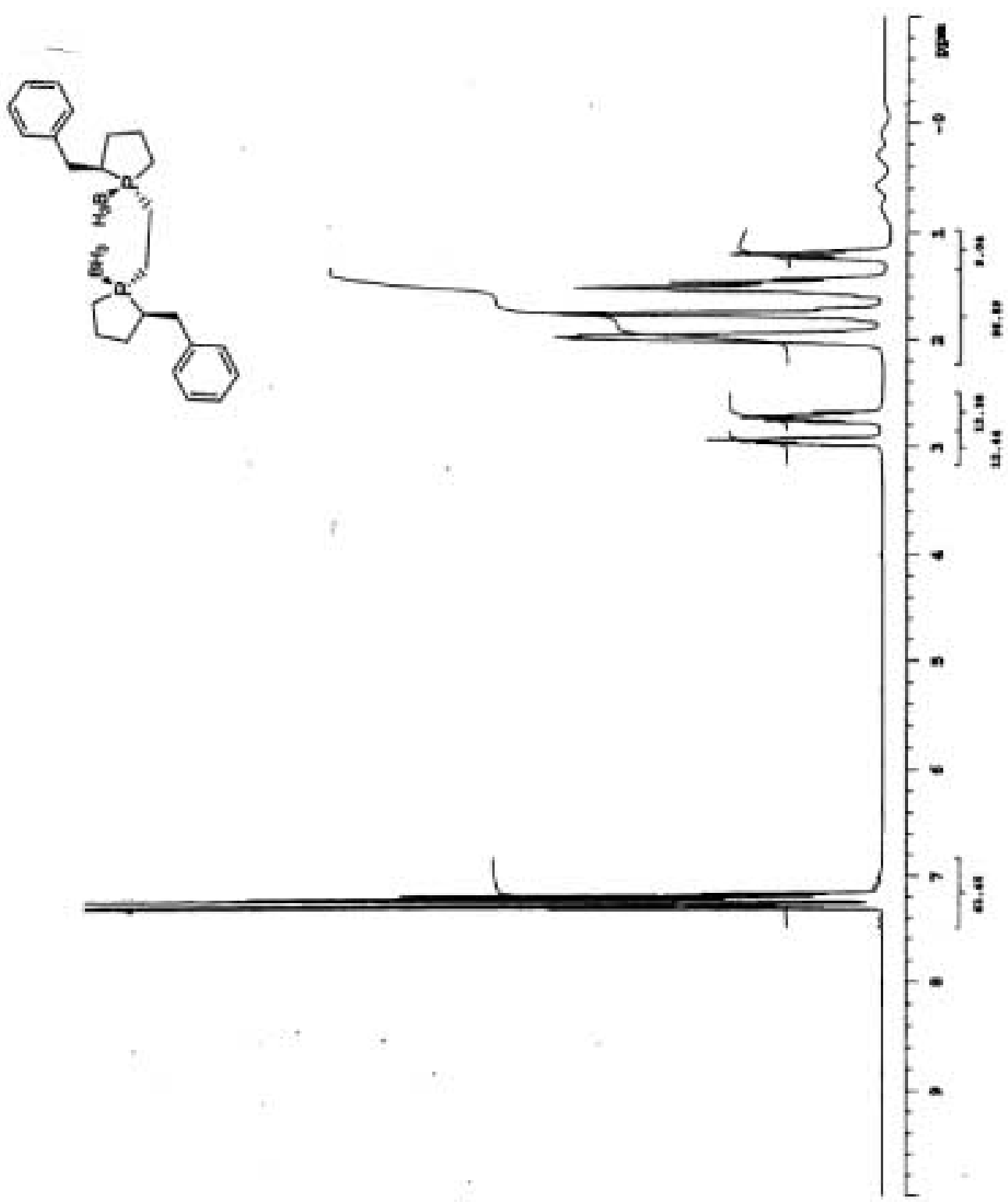

Figure 12S. ${ }^{1} \mathrm{H}$ NMR of compound $25\left(400 \mathrm{MHz}, \mathrm{CDCl}_{3}\right)$. 

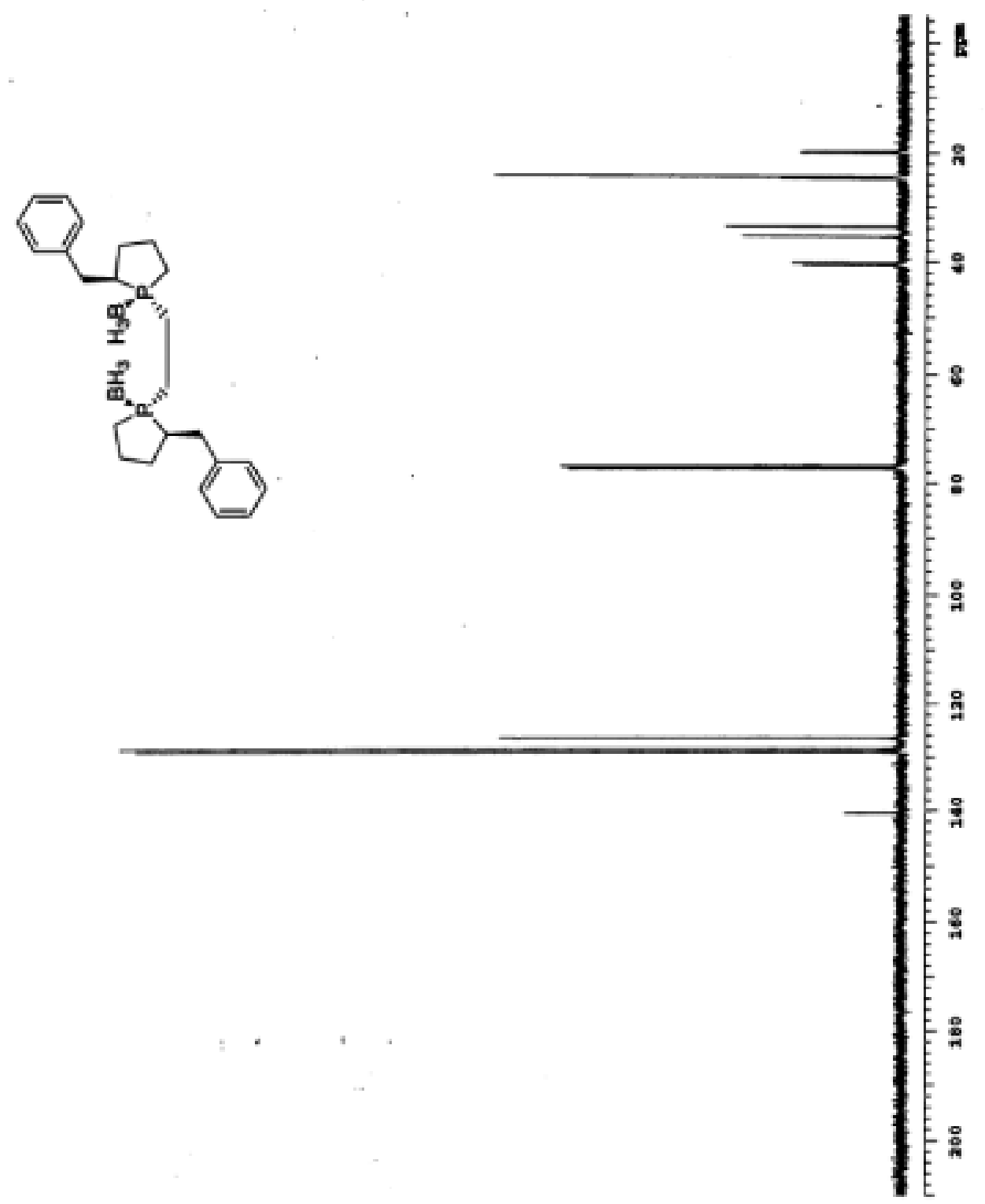

Figure 13S. ${ }^{13} \mathrm{C}$ NMR of compound $25\left(100 \mathrm{MHz}, \mathrm{CDCl}_{3}\right)$. 


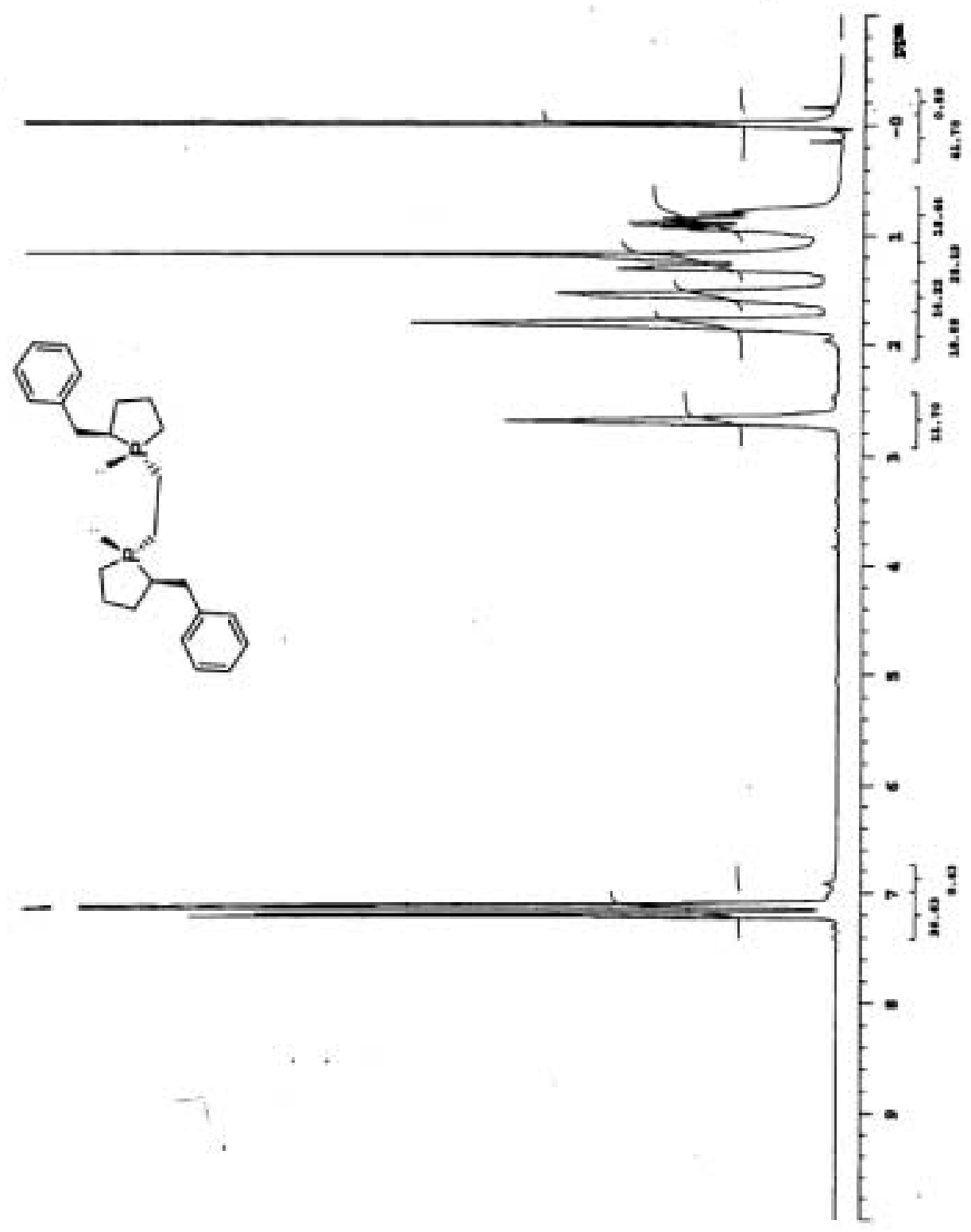

Figure 14S. ${ }^{1} \mathrm{H}$ NMR of compound $19\left(400 \mathrm{MHz}, \mathrm{CDCl}_{3}\right)$. 


\section{Supporting Information References}

${ }^{1}$ Wolf, J.P., III; Neimann, C. Biochemistry, 1963, 2, 493.

${ }^{2}$ Vineyard, B.D.; Knowles, W.S.; Sabacky, M.J.; Bachman, G.L.; Weinkauff, D.J.; J. Am. Chem. Soc., 1977, 99, 5946.

${ }^{3}$ Polniaszek, R.P.; Foster, A.L.; J. Org. Chem. 1991, 56, 3137. 